\title{
Search for topological defect dark matter using the global network of optical magnetometers for exotic physics searches (GNOME)
}

\section{Samer Afach}

Johannes Gutenberg-Universität

\section{Ben Buchler}

Australian National University https://orcid.org/0000-0002-2852-7483

Dmitry Budker

University of California at Berkeley https://orcid.org/0000-0002-7356-4814

Conner Dailey

University of Nevada https://orcid.org/0000-0003-2488-3461

\section{Andrei Derevianko}

University of Nevada Reno https://orcid.org/0000-0002-7105-4853

\section{Vincent Dumont}

Lawrence Berkeley National Laboratory

\section{Nataniel L. Figueroa}

Johannes Gutenberg-Universität Mainz

Ilja Gerhardt

University of Stuttgart

\section{Zoran Grujic}

University of Belgrade

\section{Hong Guo}

Peking University

\section{Chuanpeng Hao}

University of Science and Technology of China

\section{Paul Hamilton}

University of California

\section{Morgan Hedges}

The Australian National University

\section{Derek Jackson Kimball}

California State University - East Bay https://orcid.org/0000-0003-2479-6034

\section{Dongok Kim}

Korea Advanced Institute of Science and Technology https://orcid.org/0000-0002-4097-2889

\section{Sami Khamis}


University of California

Thomas Kornack

Twinleaf LLC

\section{Victor Lebedev}

University of Fribourg

\section{Zheng-Tian Lu}

University of Science and Technology of China

\section{Hector Masia-Roig ( $\square$ hemasiar@uni-mainz.de )}

Johannes Gutenberg-Universität

\section{Madeline Monroy}

University of California - Berkeley

\section{Mikhail Padniuk}

Jagiellonian University in Krakow https://orcid.org/0000-0002-8709-143X

\section{Christopher Palm}

California State University - East Bay

\section{Sun Yool Park}

JILA, NIST and University of Colorado Boulder

\section{Karun Paul}

The Australian National University

\section{Alexander Penaflor}

California State University - East Bay

\section{Xiang Peng}

Peking University

\section{Maxim Pospelov}

University of Minnesota

\section{Rayshaun Preston}

California State University - East Bay

\section{Szymon Pustelny}

Jagiellonian University in Krakow

\section{Theo Scholtes}

Leibniz Institute of Photonic Technology

\section{Perrin Segura}

Harvard University

\section{Yannis Semertzidis}

Institute for Basic Science https://orcid.org/0000-0001-7941-6639

\section{Dong Sheng}

University of Science and Technology of China

\section{Yun Chang Shin}

Institute for Basic Science 


\section{Joseph Smiga}

Johannes Gutenberg-Universität Mainz https://orcid.org/0000-0002-5492-5485

Jason Stalnaker

Oberlin College

Ibrahim Sulai

Bucknell University

Dhruv Tandon

Oberlin College

Tao Wang

Princeton University

Antoine Weis

University of Fribourg

Arne Wickenbrock

Johannes Gutenberg-Universität Mainz

Tatum Wilson

California State University - East Bay

\section{Teng Wu}

Peking University

David Wurm

Technische Universität München https://orcid.org/0000-0003-1287-7427

\section{Wei Xiao}

Peking University

\section{Yucheng Yang}

Peking University https://orcid.org/0000-0003-4568-3450

\section{Dongrui Yu}

Peking University

Jianwei Zhang

Peking University

\section{Article}

Keywords: Transient Signals, Exotic Fields, Earth-scale Detector, Ultralight Bosons, Axion-like Particles, Dark Matter Scenarios,

Posted Date: April 7th, 2021

DOI: https://doi.org/10.21203/rs.3.rs-298059/v1

License: (9) This work is licensed under a Creative Commons Attribution 4.0 International License. Read Full License 
Version of Record: A version of this preprint was published at Nature Physics on December 7th, 2021. See the published version at https://doi.org/10.1038/s41567-021-01393-y. 


\section{Search for topological defect dark matter using the global network of optical magnetometers for exotic physics searches (GNOME)}

Samer Afach, ${ }^{1,2}$ Ben C. Buchler, ${ }^{3}$ Dmitry Budker, ${ }^{1,2,4}$ Conner Dailey, ${ }^{5,}{ }^{*}$ Andrei Derevianko, ${ }^{5}$ Vincent Dumont, ${ }^{6}$ Nataniel L. Figueroa, ${ }^{1,2}$ Ilja Gerhardt,${ }^{7}$ Zoran D. Grujić, ${ }^{8,9}$ Hong Guo,${ }^{10}$ Chuanpeng Hao, ${ }^{11}$ Paul S. Hamilton, ${ }^{12}$ Morgan Hedges, ${ }^{3}$ Derek F. Jackson Kimball, ${ }^{13}$ Dongok Kim, $,{ }^{14}, 15$ Sami Khamis, ${ }^{12}$ Thomas Kornack, ${ }^{16}$ Victor Lebedev, ${ }^{9}$ Zheng-Tian Lu, ${ }^{17}$ Hector Masia-Roig,,${ }^{1,2, \dagger}$ Madeline Monroy, ${ }^{4,13}$ Mikhail Padniuk, ${ }^{18}$ Christopher A. Palm,${ }^{13}$ Sun Yool Park,${ }^{19,}{ }^{\ddagger}$ Karun V. Paul,${ }^{3}$ Alexander Penaflor, ${ }^{13}$ Xiang Peng, ${ }^{10}$ Maxim Pospelov, ${ }^{20,21}$ Rayshaun Preston, ${ }^{13}$ Szymon Pustelny, ${ }^{18}$ Theo Scholtes $,{ }^{9}, 22$ Perrin C. Segura, ${ }^{19}, \S$ Yannis K. Semertzidis, ${ }^{14,15}$ Dong Sheng, ${ }^{11}$ Yun Chang Shin, ${ }^{14}$ Joseph A. Smiga,,${ }^{1,2}$, Jason E. Stalnaker, ${ }^{19}$ Ibrahim Sulai, ${ }^{23}$ Dhruv Tandon, ${ }^{19}$ Tao Wang, ${ }^{24}$ Antoine Weis, ${ }^{9}$ Arne Wickenbrock,,${ }^{1,2}$ Tatum Wilson, ${ }^{13}$ Teng Wu ${ }^{10}$ David Wurm, ${ }^{25}$ Wei Xiao, ${ }^{10}$ Yucheng Yang, ${ }^{10}$ Dongrui Yu, ${ }^{10}$ and Jianwei Zhang ${ }^{10}$

${ }^{1}$ Johannes Gutenberg-Universität Mainz, 55128 Mainz, Germany

${ }^{2}$ Helmholtz-Institut Mainz, GSI Helmholtzzentrum für Schwerionenforschung, 55128 Mainz, Germany

${ }^{3}$ Centre for Quantum Computation and Communication Technology,

Research School of Physics, The Australian National University, Acton 2601, Australia

${ }^{4}$ Department of Physics, University of California at Berkeley, Berkeley, California 94720-7300, USA

${ }^{5}$ Department of Physics, University of Nevada, Reno, Nevada 89557, USA

${ }^{6}$ Computational Research Division, Lawrence Berkeley National Laboratory, Berkeley, CA 94720, USA

${ }^{7}$ Institute for Quantum Science and Technology (IQST), 3rd Institute of Physics, and Max Planck Institute for Solid State Research, D-70569 Stuttgart, Germany

${ }^{8}$ Institute of Physic Belgrade, University of Belgrade, 11080 Belgrade, Serbia

${ }^{9}$ Physics Department, University of Fribourg, Chemin du Musée 3, CH-1700 Fribourg, Switzerland

${ }^{10}$ State Key Laboratory of Advanced Optical Communication Systems and Networks, Department of Electronics, and Center for Quantum Information Technology, Peking University, Beijing 100871, China

${ }^{11}$ Department of Precision Machinery and Precision Instrumentation,

University of Science and Technology of China, Hefei 230026, P. R. China

${ }^{12}$ Department of Physics and Astronomy, University of California, Los Angeles, California 90095, USA

${ }^{13}$ Department of Physics, California State University - East Bay, Hayward, California 94542-3084, USA

${ }^{14}$ Center for Axion and Precision Physics Research, IBS, Daejeon 34051, Republic of Korea

${ }^{15}$ Department of Physics, KAIST, Daejeon 34141, Republic of Korea

${ }^{16}$ Twinleaf LLC, 300 Deer Creek Drive, Plainsboro, NJ 08536, USA

${ }^{17}$ Hefei National Laboratory for Physical Sciences at the Microscale, University of Science and Technology of China, Hefei 230026, P. R. China

${ }^{18}$ Institute of Physics, Jagiellonian University in Krakow, prof. Stanistawa Eojasiewicza 11, 30-348, Kraków, Poland

${ }^{19}$ Department of Physics and Astronomy, Oberlin College, Oberlin, OH 44074, USA

${ }^{20}$ School of Physics and Astronomy, University of Minnesota, Minneapolis, MN 55455, USA

${ }^{21}$ William I. Fine Theoretical Physics Institute, School of Physics and Astronomy, University of Minnesota, Minneapolis, MN 55455, USA

${ }^{22}$ Leibniz Institute of Photonic Technology, Albert-Einstein-Straße 9, D-07745 Jena, Germany

${ }^{23}$ Department of Physics and Astronomy, One Dent Drive,

Bucknell University, Lewisburg, Pennsylvania 17837, USA

${ }^{24}$ Department of Physics, Princeton University, Princeton, New Jersey, 08544, USA

${ }^{25}$ Technische Universität München, 85748 Garching, Germany

(Dated: February 26, 2021)

Results are reported from the first full-scale search for transient signals from exotic fields of astrophysical origin using data from a newly constructed Earth-scale detector: the Global Network of Optical Magnetometers for Exotic physics searches (GNOME). Data collected by the GNOME consist of correlated measurements from optical atomic magnetometers located in laboratories all over the world. GNOME data are searched for patterns of signals propagating through the network consistent with exotic fields composed of ultralight bosons such as axion-like particles (ALPs). Analysis of data from a continuous month-long operation of the GNOME finds no statistically significant signals consistent with those expected due to encounters with topological defects (axion domain walls), placing new experimental constraints on such dark matter scenarios. 
47 The nature of dark matter, an invisible substance com${ }_{48}$ prising over $80 \%$ of the mass of the universe [1-3], is one 49 of the most profound mysteries of modern physics. Al${ }_{50}$ though evidence for the existence of dark matter comes 51 from its gravitational interactions, unraveling its nature 52 likely requires observing non-gravitational interactions 53 between dark matter and ordinary matter [4]. One of 54 the leading hypotheses is that dark matter consists of 55 ultralight bosons such as axions [5-7] or axion-like par56 ticles (ALPs) [8-10]. Axions and ALPs arise from spon${ }_{57}$ taneous symmetry breaking at an unknown energy scale ${ }_{58} f_{\mathrm{SB}}$, which, along with their mass $m_{a}$, determines many 59 of their physical properties.

${ }_{60}$ ALPs can form stable, macroscopic field configurations ${ }_{61}$ in the form of topological defects [11-14] or composite ${ }_{62}$ objects bound together by self-interactions such as bo${ }_{63}$ son stars [15-17]. Such ALP field configurations could ${ }_{64}$ concentrate the dark matter density into many distinct, ${ }_{65}$ compact spatial regions that are small compared to the ${ }_{66}$ galaxy but much larger than the Earth. In such scenar${ }_{67}$ ios, Earth-bound detectors would only be able to measure ${ }_{68}$ signals associated with dark matter interactions on occa${ }_{69}$ sions when the Earth passes through such a dark-matter 70 object. It turns out that there is a wide range of param${ }_{71}$ eter space, consistent with observations, for which such 72 dark-matter objects can have the required size and abun73 dance such that the characteristic time between encoun74 ters could be on the order of one year or less [13, 17, 18]. 75 This opens up the possibility of searches with terrestrial 76 detectors. Here we present the results of such a search for 77 ALP domain walls, a class of topological defects which 78 can form between regions of space with different vacua 79 of an ALP field [11-14]. ${ }^{1}$

80 Since ALPs can interact with atomic spins [4], the pas${ }_{81}$ sage of Earth through an ALP domain wall affects atomic 82 spins similarly to a transient magnetic field pulse $[13,17]$. ${ }_{83}$ Considering a linear coupling between the ALP field gra${ }_{84}$ dient $\boldsymbol{\nabla} a(\boldsymbol{r}, t)$ and atomic spin $\boldsymbol{S}$, the interaction Hamil-

* Currently: University of Waterloo, Department of Physics and Astronomy, N2L 3G1, Ontario, CA

$\dagger$ hemasiar@uni-mainz.de

$\ddagger$ Currently: JILA, NIST and University of Colorado, and Department of Physics, University of Colorado, Boulder Colorado 80309-0440, USA

$\S$ Currently: Department of Physics, Harvard University, Cambridge, MA 02138

I jsmiga@uni-mainz.de

1 A different type of bosonic field configuration is the Q-ball [19, $20]$ which is spherically symmetric having nearly constant field magnitude in the bulk with a rapid fall off at the boundaries. It is another possible dark matter candidate. We note that the signal pattern produced in GNOME for sufficiently large Q-balls with couplings to atomic spins can be similar to that of ALP domain walls [17].
85 tonian can be written as

$$
H_{\text {lin }}=-(\hbar c)^{3 / 2} \frac{\xi}{f_{\mathrm{SB}}} \frac{\boldsymbol{S}}{\|S\|} \cdot \nabla a(\boldsymbol{r}, t),
$$

${ }_{86}$ where $\hbar$ is the reduced Planck's constant, $c$ is the speed ${ }_{87}$ of light, $\boldsymbol{r}$ is the position of the spin, $t$ is the time, and ${ }_{88} f_{\mathrm{SB}} / \xi \equiv f_{\text {int }}$ is the coupling constant in units of energy ${ }_{89}$ described with respect to the symmetry-breaking scale ${ }_{90} f_{\mathrm{SB}}[21]$, where $\xi$ is unitless. In most theories, the cou${ }_{91}$ pling constants $f_{\text {int }}$ describing the interaction between ${ }_{92}$ Standard Model fermions and the ALP field are propor${ }_{93}$ tional to $f_{\mathrm{SB}}$; however, $f_{\text {int }}$ can differ between electrons, ${ }_{94}$ neutrons, and protons by model-dependent factors that ${ }_{95}$ can be significant in some cases $[4,8]$.

96 In analogy with Eq. (1), the Zeeman Hamiltonian de${ }_{97}$ scribing the interaction of a magnetic field $\boldsymbol{B}$ with an 98 atomic spin $\boldsymbol{S}$ can be written as

$$
H_{\mathrm{Z}}=-\gamma \boldsymbol{S} \cdot \boldsymbol{B},
$$

99 where $\gamma$ is the gyromagnetic ratio. Since Eqs. (1) and (2) 100 have the same structure, the gradient of the ALP field 101 can be treated as a pseudo-magnetic field. An important 102 distinction between the ALP-spin interaction [Eq. (1)] 103 and the Zeeman interaction [Eq. (2)] is that while $\gamma$ tends 104 to scale inversely with the fermion mass, no such scaling 105 of the ALP-spin interaction is expected [4].

The amplitude, direction, and duration of the pseudo${ }_{107}$ magnetic field pulse associated with the transit of the ${ }_{108}$ Earth through an ALP domain wall depends on many 109 unknown parameters such as the energy density stored in 110 the ALP field, the coupling constant $f_{\text {int }}$, the thickness 111 of the domain wall, and the relative velocity $\boldsymbol{v}$ between ${ }_{112}$ Earth and the domain wall. The dynamical parameters, 113 such as the velocities of the dark matter objects, are ex114 pected to randomly vary from encounter-to-encounter. ${ }_{115}$ We assume that they are described by the Standard Halo 116 Model for virialized dark matter [22]. Furthermore, the 117 abundance of domain walls in the galaxy is limited by ${ }_{118}$ physical constants, $m_{a}$ and $f_{\mathrm{SB}}$, as these determine the ${ }_{119}$ energy contained in the wall and the total energy of all 120 domain walls is constrained by estimates of the local dark 121 matter density [23]. The expected temporal form of the 122 pseudo-magnetic field pulse can depend both on the the${ }_{123}$ oretical model describing the ALP domain wall as well as ${ }_{124}$ particular details of the terrestrial encounter such as the 125 orientation of the Earth. The relationships between these ${ }_{126}$ parameters and characteristics of the pseudo-magnetic ${ }_{127}$ field pulses searched for in our analysis are discussed in ${ }_{128}$ Appendix A and Refs. [13, 17, 21].

${ }_{129}$ The Global Network of Optical Magnetometers for Ex130 otic physics searches (GNOME) is a worldwide network 131 searching for correlated signals heralding beyond-the${ }_{132}$ Standard-Model physics which currently consists of more 133 than a dozen optical atomic magnetometers, with sta134 tions in Europe, North America, Asia, the Middle East, 135 and Australia. A schematic of a domain-wall encounter 136 with the GNOME with stations used in this study is 


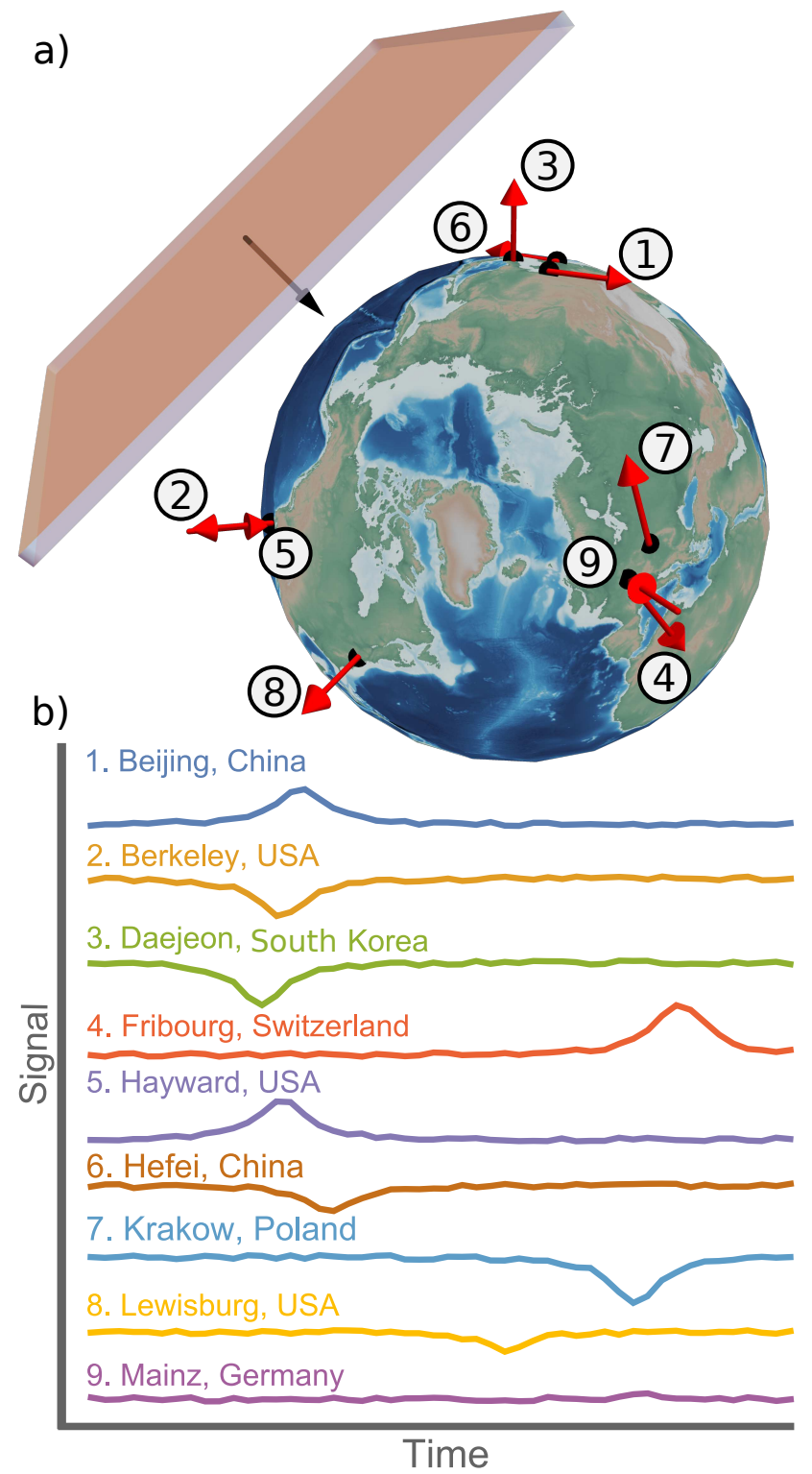

Figure 1. (a) Visualization of an ALP domain wall crossing the Earth. The red arrows indicate the position and sensitive axes of the GNOME magnetometers during Science Run 2 (Sec. I). (b) Simulation of the signals expected to be observed from a domain-wall crossing.

${ }_{137}$ shown in Fig. 1. The measurements from the magne138 tometers are recorded with data-acquisition systems, syn139 chronized to the Global Positioning System (GPS) time, 140 and uploaded to servers located in Mainz, Germany, and ${ }_{141}$ Daejeon, South Korea. Descriptions of the operational 142 principles and characteristics of GNOME magnetome${ }_{143}$ ters are presented in Sec. IV and Ref. [24], details on 144 the GNOME data acquisition system are discussed in ${ }_{145}$ Ref. [25] (see also Table I).

146 The active field sensor at the heart of every GNOME 147 magnetometer is an optically pumped and probed gas 148 of alkali atoms. Magnetic fields are measured through
149 variations in the Larmor spin precession of the optically 150 polarized atoms. The vapor cells containing the alkali 151 atoms are placed inside multi-layer magnetic shielding 152 systems which reduce background magnetic noise by or153 ders of magnitude [26] while retaining sensitivity to ex154 otic spin-couplings between ALP dark matter and atomic 155 nuclei. $^{2}$ Since all GNOME magnetometers presently use 156 atoms whose nuclei have a valence proton, the signal am${ }_{157}$ plitudes measured by the GNOME due to an ALP-spin ${ }_{158}$ interaction are proportional to the relative contribution 159 of the proton spin to the nuclear spin (as discussed in ${ }_{160}$ Appendix B and Ref. [28]). This pattern of signal ampli${ }_{161}$ tudes [Eq. (1)] can be characterized by a pseudo-magnetic ${ }_{162}$ field $B_{j}$ measured with sensor $j$ :

$$
B_{j}=\frac{\sigma_{j} \eta_{j}}{g_{F, j}} \mathcal{B}_{\mathrm{p}}
$$

163 where

$$
\mathcal{B}_{\mathrm{p}}(\boldsymbol{r}, t)=(\hbar c)^{3 / 2} \frac{2 \xi}{\mu_{B} f_{\mathrm{SB}}} \nabla a(\boldsymbol{r}, t)
$$

164 is the normalized pseudo-magnetic field describing the ${ }_{165}$ effect of the ALP domain wall on proton spins and $\mu_{B}$ 166 is the Bohr magneton. The ratio between the Landé g${ }_{167}$ factor and the effective proton spin $\left(g_{F, j} / \sigma_{j}\right)$ accounts for 168 the specific proton-spin coupling in the respective sensor. 169 This ratio depends on the atomic and nuclear structure 170 as well as details of the magnetometry scheme, see Ap171 pendix B. Since each GNOME magnetometer measures 172 the projection of the field along a particular sensitive 173 axis, the factor $\eta_{j}$ is introduced to account for the direc174 tional sensitivity. This factor, given by the cosine of the 175 angle between $\mathcal{B}_{\mathrm{p}}$ and the sensitive axes, takes on values 176 between \pm 1 .

In spite of the unknown properties of a particular 178 terrestrial encounter with an ALP domain wall, the ${ }_{179}$ GNOME would measure a recognizable global pattern 180 of the associated pseudo-magnetic field pulse amplitudes 181 described by Eq. (3), as illustrated in Fig. 1b. The as182 sociated pseudo-magnetic field pulses would point along 183 a common axis, have the same duration, and exhibit a 184 characteristic timing pattern. The data-analysis algo185 rithm used in the present work to search for ALP domain 186 walls is described in Sec. IV and Ref. [29]. The algo${ }_{187}$ rithm searches for a characteristic signal pattern across ${ }_{188}$ the GNOME having properties consistent with passage 189 of the Earth through an ALP domain wall. Separate 190 analyses to search for transient oscillatory signals associ191 ated with boson stars [17] and bursts of exotic low-mass 192 fields (ELFs) from cataclysmic astrophysical events [30] 193 are presently underway.

Here we report the first results of a dark matter search 195 with the GNOME: a search for transient couplings of

\footnotetext{
2 Signals due to ALP interactions with electron spins can be counteracted by effects from interaction between the ALP field and electron spins in the magnetic shielding material [27].
} 
196 atomic spins to macroscopic dark-matter objects, and 251 time, the amplitudes measured by each magnetometer 197 therefore demonstrate the ability of the GNOME to ex- 252 are fit to the ALP domain-wall crossing model described 198 plore parameter space previously unconstrained by direct 253 in Ref. [29]. As a result, estimations for signal magnitude 199 laboratory experiments. Searches for macroscopic dark- 254 and domain-wall direction, along with associated uncer200 matter objects based on similar ideas were carried out 255 tainties, are obtained for each measurement time and all 201 using atomic clock networks [18, 31-33], and there are a 256 lattice velocities. The magnitude-to-uncertainty ratio of 202 number of experimental proposals utilizing other sensor 257 an event is given by the ratio between the signal magni203 networks [34-38]. All of these networks are sensitive to 258 tude and its associated uncertainty. 204 bosonic dark matter with a scalar coupling to Standard 205 Model particles [4]. The GNOME is sensitive to a differ206 ent class of dark matter: bosons with pseudoscalar cou207 plings to Standard Model particles. Pseudoscalar bosonic 208 dark matter generally produces no observable effects in 209 clock networks [4] but does couple to atomic spins via the 210 interaction described by Eq. (1). Thus the GNOME is 211 sensitive to a distinct, so far mostly unconstrained, class 212 of interactions as compared to other sensor networks.

\section{RESULTS}

${ }_{214}$ There have been four GNOME Science Runs between 2152017 and 2020 as discussed in Sec. IV. Here we analyze 216 the data from Science Run 2, which had comparatively 217 good overall noise characteristics and consistent network ${ }_{218}$ operation (as seen in Fig. 5). Nine magnetometers took 219 part in Science Run 2 that spanned from 29 November 2202017 to 22 December 2017. The characteristics of the 221 magnetometers are summarized in Table I.

222 Before the data are searched for evidence of domain${ }_{223}$ wall signatures, they are preprocessed by applying a 224 rolling average, high-pass filters, and notch filters to the 225 raw data. The averaging enhances the signal-to-noise ra226 tio for certain pulse durations, avoids complications aris227 ing from different magnetometers having different band228 widths, and reduces the amount of data to be analyzed. 229 The high-pass and notch filters reduce the effects of long230 term drifts and noisy frequency bands. We refer to the fil231 tered and rolling-averaged data set as the "search data."

232 The search data are examined for evidence of collective 233 signal patterns corresponding to planes with uniform, 234 non-zero thickness, crossing Earth at constant veloci235 ties. The imprinted pattern of amplitudes depends on the 236 domain-wall crossing velocity [29]. As noted in Sec. I, we 237 assume that the domain-wall-velocity probability density 238 function follows the Standard Halo Model for virialized 239 dark matter. The signature of a domain-wall crossing 240 the magnetometer network depends on the perpendicu${ }_{241}$ lar component to the domain-wall plane of the relative ${ }_{242}$ velocity between the Earth and the domain wall, $\boldsymbol{v}_{\perp}$. A
${ }_{243}$ lattice of points in velocity space is constructed such that 244 the search algorithm covers $97.5 \%$ of the velocity proba245 bility density function. The algorithm scans over the ve-
246 locity lattice and, for every velocity, the data from each 247 magnetometer are appropriately time-shifted so that the 248 signals in different magnetometers from a hypothetical 249 domain-wall crossing with the given velocity occur at the 250 same time. For each velocity and at each measurement
The search algorithm uses two different tests to eval260 uate if a given event is likely to have been produced 261 by an ALP domain-wall-crossing: a domain-wall model 262 test and a directional-consistency test [29]. The domain263 wall model test evaluates whether the event amplitudes 264 measured by the GNOME magnetometers match the sig265 nal amplitudes predicted by the ALP domain-wall cross266 ing model, and is quantified by the $p$-value as discussed 267 in Sec. IV and Ref. [29]. The directional-consistency 268 test checks the agreement between the direction of the 269 scanned velocity and the estimated domain wall direc270 tion, and is quantified by the angle between the two di271 rections normalized by the angle between adjacent lattice 272 velocities.

273 In order to evaluate the detection probability of the 274 search algorithm, a well-characterized data set that in275 cludes domain-wall-crossing signals with known proper276 ties is required. For this purpose, we generate a back277 ground data set by randomly time-shuffling the search 278 data so that the relative timing of measurements from 279 different GNOME stations is shifted by amounts so large 280 that no true-positive events could occur. By repeating 281 the process of time shuffling, the length of the back282 ground data can be made to far exceed the search data. 283 This method is used to generate background data with 284 noise characteristics closely reproducing those of the 285 search data [39]. A set of pseudo-magnetic field pulses 286 matching the expected amplitude and timing pattern 287 produced by the passages of Earth through ALP domain 288 walls are inserted into the background data to create the 289 test data. The test data are used to evaluate the true290 positive probability for the search algorithm for different 291 thresholds on the $p$-value and the normalized angular dif292 ference. The detection probability as a function of the 293 thresholds is studied in order to find a combination of 294 thresholds that ensures the detection of $97.5 \%$ of the in295 serted domain walls (see Sec. IV and Fig. 6). This results 296 in an overall detection efficiency $\epsilon \geq 95 \%$ for the search 297 algorithm, considering both the incomplete velocity lat298 tice coverage and the detection probability.

The search data are analyzed for domain-wall encoun300 ters using the algorithm presented in Ref. [29]. The 301 cumulative distribution of candidate events as a func302 tion of their magnitude-to-uncertainty ratio is shown as 303 a solid green line in Fig. 2. The background data are 304 analyzed in the same way as the search data to estimate 305 the expected number of background events during Sci306 ence Run 2. Time-shuffling is used to create 10.7 years 307 of background data. The number of candidate events 308 measured in the background data re-scaled to the dura- 


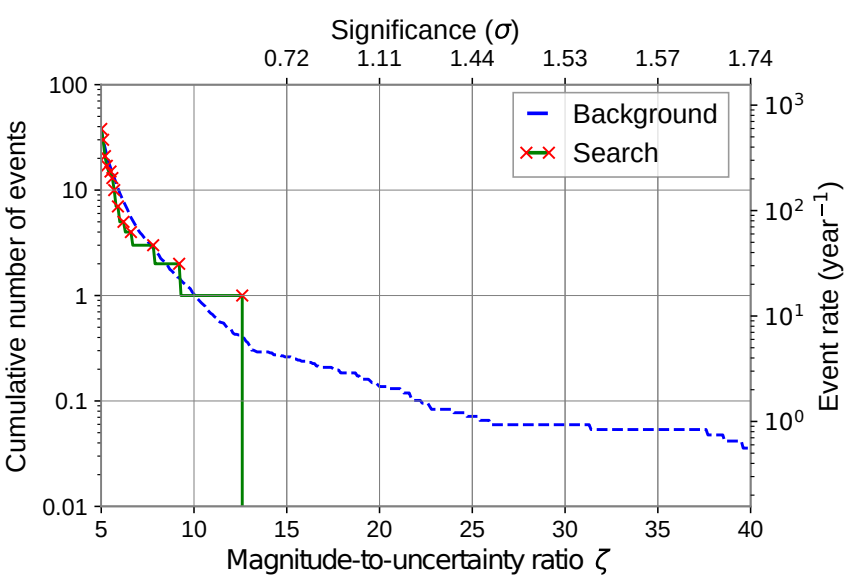

Figure 2. The blue dashed line represents the number events expected from the background in the twenty-three days of data from Science Run 2. 10.7 years of time-shuffled data are used to evaluate the background. The solid green line represents the number of events measured in Science Run 2. The red crosses indicate at which magnitude-to-uncertainty ratio new events are found. The upper axis indicates the statistical significance in units of Gaussian standard deviations of finding one event in the search data. The event with greatest magnitude-to-uncertainty ratio is found at 12.6.

309 tion of Science Run 2 is shown as a dashed blue line in 310 Fig. 2. The significance of a candidate event in the search 311 data is given by the probability of detecting one or more 312 background events at a magnitude-to-uncertainty ratio 313 above that of the candidate event [see Eq. (10)]. The 314 probability of finding a number of candidate events in 315 the background data follows Poisson statistics.

316 The candidate event in the search data with the largest 317 magnitude-to-uncertainty ratio $(=12.6)$ had a signifi318 cance of less than one sigma. Therefore, we find no ev319 idence of an ALP domain-wall crossing during Science 320 Run 2.

321 In order to evaluate the domain-wall characteristics ex322 cluded by this result, the observable domain-wall cross323 ing parameters above 12.6 magnitude-to-uncertainty ra324 tio during Science Run 2 are determined. The GNOME 325 has nonuniform directional sensitivity [29]; we conserva326 tively estimate the network sensitivity assuming the do327 main wall comes from the least-sensitive direction. Fig- ${ }^{356}$ ${ }_{328}$ ure 3 shows the active time $\tilde{T}\left(\Delta t, \mathcal{B}_{\mathrm{p}}^{\prime}\right)$, i.e., how long 329 the network was sensitive to domain-walls as a function ${ }^{357}$ 330 of pseudo-magnetic field-pulse-magnitude sensitivity, $\mathcal{B}_{\mathrm{p}}^{\prime}$, 331 and pulse duration, $\Delta t$. A signal with pseudo-magnetic ${ }_{332}$ field magnitude $\mathcal{B}_{\mathrm{p}}$ produces a magnitude-to-uncertainty ${ }_{333}$ ratio of $\zeta=\mathcal{B}_{\mathrm{p}} / \mathcal{B}_{\mathrm{p}}^{\prime}$. The characteristic shape of the sen- 360 334 sitive region is a result of the filtering and averaging of 362 mass, $m_{a}$, the domain-wall energy density in the Milky 335 the raw data as described in Sec. IV. Averaging reduces 363 Way, $\rho_{\mathrm{DW}}$, the typical relative domain-wall speed $\bar{v}$ given 336 the sensitivity of the search data to short pulse durations 364 by the Standard Halo Model, and the symmetry break${ }_{337}$ and high-pass filtering suppresses sensitivity for long $\Delta t .365 \mathrm{ing}$ scale, $f_{\mathrm{SB}}$. The region of parameter space to which 338 The GNOME sensitivity varies in time as the number of 366 GNOME is sensitive is defined by the ALP parameters 339 active GNOME magnetometers recording data and their 367 expected to produce signals above 12.6 magnitude-to340 background noise change. The active time, $\tilde{T}\left(\Delta t, \mathcal{B}_{\mathrm{p}}^{\prime}\right)$, 368 uncertainty ratio with rates $r \geq R_{90 \%}$ during Science

Figure 3. Amount of time $\tilde{T}$ that the GNOME is sensitive to domain walls with a given duration $\Delta t$ and normalized pseudo-magnetic field magnitude sensitivity $\mathcal{B}_{\mathrm{p}}^{\prime}$ throughout Science Run 2, the magnitude of an event needed to induce a signal with a magnitude-to-uncertainty ratio of one [see Eq. (11)]. Only the worst-case direction is considered. The plot assumes the parameters of the analysis: 20s averaging time, $1.67 \mathrm{mHz}$ first-order zero-phase Butterworth filter, and $50 \mathrm{~Hz}$ and $60 \mathrm{~Hz}$ zero-phase notch filters with a $Q$-factor of 60 .

${ }_{341}$ can be used to constrain ALP domain-wall parameter 342 space as discussed in Appendix A.

${ }_{343}$ If one assumes a probability distribution for the num344 ber of domain-wall encounters, an upper bound on the ${ }_{345}$ rate $R_{C}$ of such encounters can be calculated with a con346 fidence level $C$. We assume a Poisson probability dis347 tribution for the domain-wall crossings. Since the ex348 cess number of events in the search data as compared 349 to the background data was not statistically significant, 350 the upper bound on the observable rate is given by the 351 probability of measuring no events during the effective 352 time [40]. Note that since $\tilde{T}$ depends on the parameters 353 of the domain-wall crossing, our constraint on the ob354 served rate depends on the ALP properties. We choose 355 the confidence level to be $C=90 \%$.

\section{DISCUSSION}

The analysis of the GNOME data did not find any sta35 tistically significant excess of events above background 59 during Science Run 2 that could point to the existence so of ALP domain walls, as seen in Fig. 2. The expected 1 rate of domain-wall encounters, $r$, depends on the ALP

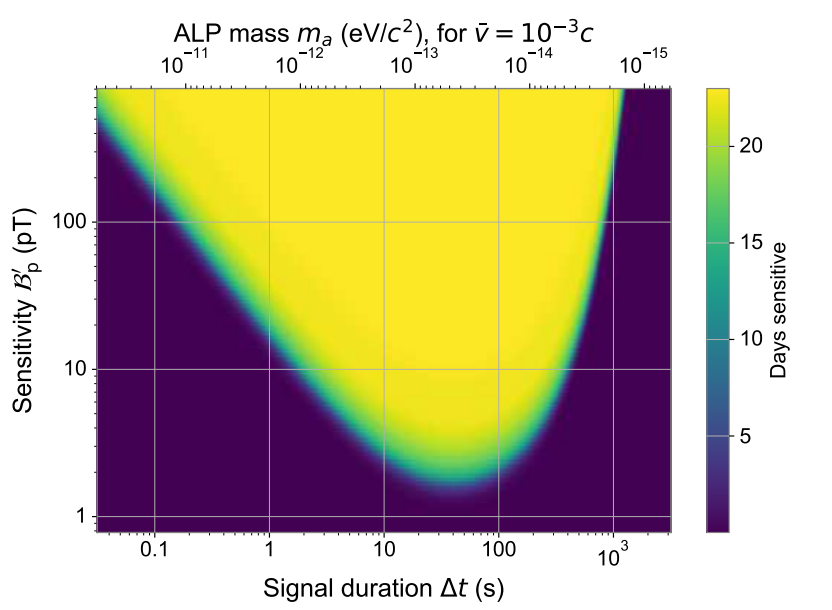

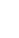


${ }_{369}$ Run 2 (see Fig. 3). Based on the null result of our search, 410 the coupling constant indicated by the dashed lines. The 370 the sensitive region is interpreted as excluded ALP pa- 411 upper bound of $f_{\mathrm{SB}}$ that can be observed by the net371 rameter space.

372 The ALP parameters and the phenomenological pa${ }_{412}$ work is shown in Fig. $4 \mathrm{~b}$ for different ratios $\xi \equiv f_{\mathrm{SB}} / f_{\text {int }}$. 373 rameters describing the ALP domain walls in our galaxy, 414 cut-off for low ALP mass where the corresponding field 374 namely the thickness $\Delta x$, the surface tension or energy 415 magnitude falls below the network sensitivity. Even ${ }_{375}$ per unit area $\sigma_{\mathrm{DW}}$, and the average separation $\bar{L}$ can be ${ }_{416}$ though $\mathcal{B}_{\mathrm{p}}$ increases for large $m_{a}$, the mean rate of 376 related through the ALP domain wall model described ${ }_{417}$ domain-wall encounters decreases with increasing mass 377 in Refs. [13, 21]. A full derivation of how observable ${ }_{418}$ [see Eqs. (8) and (9)]. Correspondingly, the upper limit ${ }_{378}$ parameters are related to ALP parameters is given in ${ }_{419}$ for the symmetry-breaking scale $f_{\mathrm{SB}}$ is $\propto 1 / \sqrt{m_{a}}$. Given 379 Appendix A and summarized below. 420 that no events were found, the sensitive region of ALP380 The domain-wall thickness is determined by the ALP ${ }_{421}$ domain-wall parameter space during Science Run 2 can 381 mass, and is on the order of the ALP reduced Compton ${ }^{422}$ be excluded. 382 wavelength $\lambda_{a}[21]$,

$$
\Delta x \approx 2 \sqrt{2} \lambda_{a}=2 \sqrt{2} \frac{\hbar}{m_{a} c} .
$$

383 The constant prefactor of $2 \sqrt{2}$ is obtained by approxi384 mating the spatial profile of the field-gradient magnitude 385 as a Lorentzian and defining the thickness as full width at 386 half maximum. For a given relative velocity component ${ }_{387}$ perpendicular to the domain wall $v_{\perp}$, the signal duration 388 is

$$
\Delta t=\frac{\Delta x}{v_{\perp}} \propto m_{a}^{-1} .
$$

\section{9} 390 component of dark matter. Thus with the energy density ${ }_{391} \rho_{\mathrm{DW}} \approx 0.4 \mathrm{GeV} / \mathrm{cm}^{3}$ in the Milky Way [23], the energy 392 per unit area (surface tension) in a domain wall, $\sigma_{\mathrm{DW}}$, 393 determines the average separation between domain walls, ${ }_{394} \bar{L}$. The surface tension $\sigma_{\mathrm{DW}}$ is related to the symmetry 395 breaking scale [13],

$$
\sigma_{\mathrm{DW}}=\frac{8}{\hbar^{2}} m_{a} f_{\mathrm{SB}}^{2} .
$$

396 The average domain-wall separation is then approxi397 mated by

$$
\bar{L} \approx \frac{\sigma_{\mathrm{DW}}}{\rho_{\mathrm{DW}}}=\frac{8}{\hbar^{2}} \frac{m_{a} f_{\mathrm{SB}}^{2}}{\rho_{\mathrm{DW}}},
$$

398 which results in the average domain-wall encounter rate,

$$
r=\bar{v} / \bar{L} \propto\left(m_{a} f_{\mathrm{SB}}^{2}\right)^{-1} .
$$

399 The colored region in Fig. 4a describes the symmetry 400 breaking scales up to which GNOME was sensitive with ${ }_{401} 90 \%$ confidence (as detailed in Appendix A). The param${ }_{402}$ eter space is spanned by ALP mass, maximum symme403 try breaking scale, and ratio between symmetry breaking 404 scale and coupling constant. The shape of the sensitive 405 area shown in Fig. 4a is determined by the event with the 406 largest magnitude-to-uncertainty and the characteristics ${ }_{407}$ of the preprocessing applied to the raw data.

${ }_{408}$ Figure 4b shows various cross-sections of Fig. 4a for 409 different ratios between the symmetry breaking scale and
${ }_{423}$ Our experiment explores ALP parameter space up to ${ }_{424} f_{\text {int }} \sim 3 \times 10^{5} \mathrm{GeV}$ (see Fig. 4). This goes beyond that ex${ }_{425}$ cluded by previous direct laboratory experiments search${ }_{426}$ ing for ALP-mediated exotic pseudoscalar interactions ${ }_{427}$ between protons which have shown that $f_{\text {int }} \gtrsim 300 \mathrm{GeV}$ ${ }_{428}$ over the ALP mass range probed by the GNOME [41]. ${ }_{429}$ Although astrophysical observations suggest that $f_{\text {int }} \gtrsim$ ${ }_{430} 2 \times 10^{8} \mathrm{GeV}$, there are a variety of scenarios in which ${ }_{431}$ such astrophysical constraints can be evaded [42, 43].

${ }_{432}$ Future work of the GNOME collaboration will focus 433 both on upgrades to our experimental apparatus and 434 new data-analysis strategies. One of our key goals is 435 to improve overall reliability and the duration of con436 tinuous operation of GNOME magnetometers ${ }^{3}$. Addi${ }_{437}$ tionally, magnetometers varied in their bandwidths and 438 reliability, and stability of their calibration. These chal439 lenges were addressed in Science Run 4 through a va440 riety of magnetometer upgrades and instituting daily ${ }_{441}$ worldwide test and calibration pulse sequences. How${ }_{442}$ ever GNOME suffered disruptions due to the worldwide ${ }_{443}$ COVID-19 pandemic. We plan to carry out Science ${ }_{444}$ Run 5 in 2021 to take full advantage of the improve445 ments. Furthermore, by upgrading to noble-gas-based ${ }_{446}$ comagnetometers $[44,45]$ for future science runs (Ad${ }_{447}$ vanced GNOME), we expect to significantly improve the 448 sensitivity to ALP domain walls. Additionally, GNOME 449 data can be searched for other signatures of physics be450 yond the Standard Model such as boson stars [17], relax451 ion halos [46], and bursts of exotic low-mass fields from 452 black-hole mergers [30]. In terms of the data-analysis al453 gorithm used to search for ALP domain walls, in future 454 work we aim to improve the efficiency of the scan over the 455 velocity lattice. The number of points in the velocity lat${ }_{456}$ tice to reliably cover a fixed fraction (e.g., $97.5 \%$ ) of the ${ }_{457}$ ALP velocity probability distribution grows as $(\Delta t)^{-3}$ ${ }_{458}$ [where $\Delta t$ is given by Eq. (6)]. This makes the algo459 rithm computationally intensive. We are investigating 460 a variety of new analysis approaches, such as machine461 learning-based algorithms, to address these issues.

\footnotetext{
3 The intermittent operation of some magnetometers due to technical difficulties during Science Runs 1-3 made it difficult to search for signals persisting $\gtrsim 1$ hour.
} 
a)

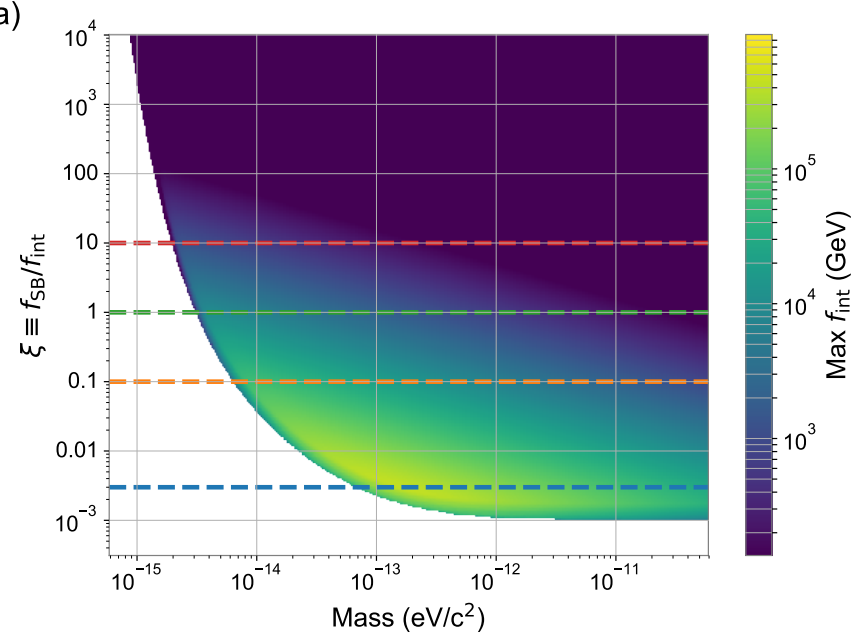

b)

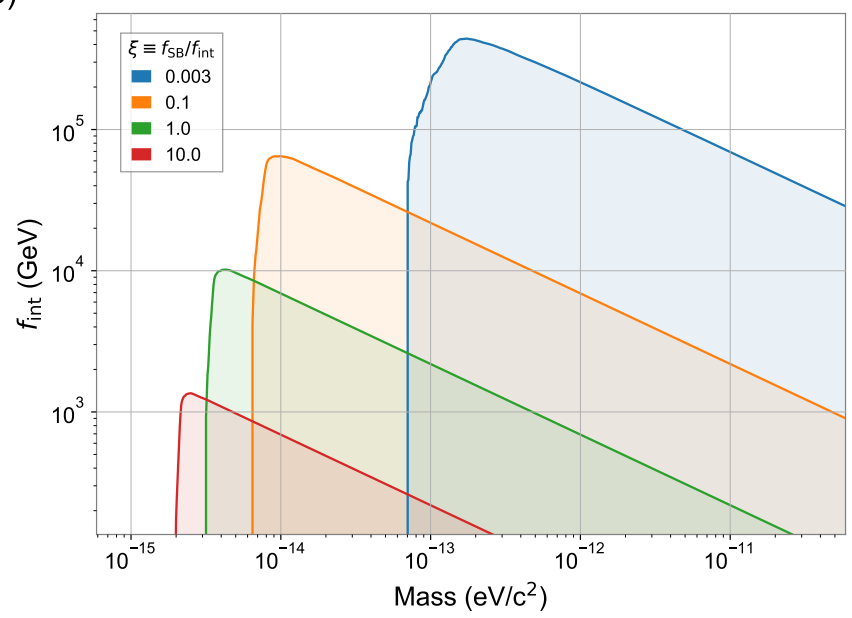

Figure 4. Parameter space of the ALP constrained by the presented analysis of Science Run 2 with $90 \%$ confidence level (see Sec. II). The relationship between ALP theory parameters and measured quantities are discussed in Sec. III and Appendix A. (a) In color, upper bound on the the interaction scale, $f_{\text {int }}$, to which the GNOME was sensitive as a function of $m_{a}$ and the ratio $\xi \equiv f_{\mathrm{SB}} / f_{\text {int }}$. (b) Cross-sections of the excluded parameter volume in (a) for different ratios $\xi$ represented by the corresponding dashed lines of the matching color in (a).

\section{METHODS}

The GNOME consists of over a dozen optical atomic 464 magnetometers, each enclosed within a multi-layer mag465 netic shield, distributed around the world [26]. GNOME 466 magnetometers are based on a variety of different atomic ${ }_{467}$ species, optical transitions, and measurement techniques: 468 some are frequency- or amplitude-modulated nonlinear 469 magneto-optical rotation magnetometers (NMOR) [47, 470 48], some are rf-driven optical magnetometers [24], while ${ }_{471}$ others are spin-exchange-relaxation-free magnetometers 472 (SERF) [49]. A detailed description and characterization ${ }_{473}$ of six GNOME magnetometers is given in Ref. [24]. A 474 summary of the properties of the GNOME magnetome475 ters active during Science Run 2 is presented in Table I.

${ }_{476}$ Each GNOME station is equipped with auxiliary 477 sensors, including accelerometers, gyroscopes, and un478 shielded magnetometers, to measure local perturbations 479 that could mimic a dark matter signal. Suspicious data 480 are flagged [24] and discarded during the analysis.

481 The number of active GNOME magnetometers during 482 the four Science Runs and the combined network noise as ${ }_{483}$ defined in Ref. [29] is shown as a function of time in Fig. 5. ${ }_{484}$ The data in Science Run 4, although they extended for a ${ }_{485}$ longer period of time, featured poorer noise characteris486 tics and consistency of operation to Science Run 2. Since ${ }_{487}$ many GNOME stations underwent upgrades in 2018 and ${ }_{488} 2019$, further characterization of Science Run 4 data is 489 needed, and results will be presented in future work.

490 Here we provide more details on the analysis proce491 dure discussed in Sec. II. It is composed of three stages to 492 identify events likely to be produced by ALP domain-wall ${ }_{493}$ crossings: preprocessing, velocity scanning, and post${ }_{494}$ selection [29]. First, in the preprocessing stage, a rolling
495 average and filters are applied to the GNOME magne496 tometer raw data which are originally recorded by the 497 GPS-synchronized data-acquisition system at a rate of 498512 samples/s [25]. The rolling average is characterized 499 by a $20 \mathrm{~s}$ time constant. Noisy frequency bands are sup500 pressed using a first-order Butterworth high-pass filter at ${ }_{501} 1.67 \mathrm{mHz}$ together with the notch filters corresponding 502 to $50 \mathrm{~Hz}$ and $60 \mathrm{~Hz}$ power line frequencies with a quality 503 factor of 60 . These filters are applied forward and back504 ward to remove phase effects. This limits the observable ${ }_{505}$ pulse properties to a frequency region to which all mag506 netometers are sensitive. Additionally, it guarantees that 507 the duration of the signal is the same in all sensors. We 508 note that these filter settings may be changed in future 509 analysis.

510 The local standard deviation around each point in 511 the magnetometer's data is determined using an itera512 tive process. Outliers are discarded until the standard 513 deviation of the data in the segment converges. The 514 local standard deviation is calculated taking 100 down515 sampled points around each data point.

516 Additionally, auxiliary measurements have shown that 517 the calibration factors used by each magnetometer to 518 convert raw data into magnetic field units experience 519 change over time due to, for example, changes in the en520 vironmental conditions. Upper limits on the calibration ${ }_{521}$ factor errors due to such drifts over the course of Science ${ }_{522}$ Run 2 have been evaluated and are listed in Table I. Cali${ }_{523}$ bration factor errors result in magnetic field measurement ${ }_{524}$ errors proportional to the magnetic field $B_{j}$. The uncer525 tainty resulting from the calibration error is later used to ${ }_{526}$ determine agreement with the domain-wall model, but ${ }_{527}$ not in the magnitude-to-uncertainty ratio estimate re${ }_{528}$ sulting from the model, since the calibration error affects 
a)

b)
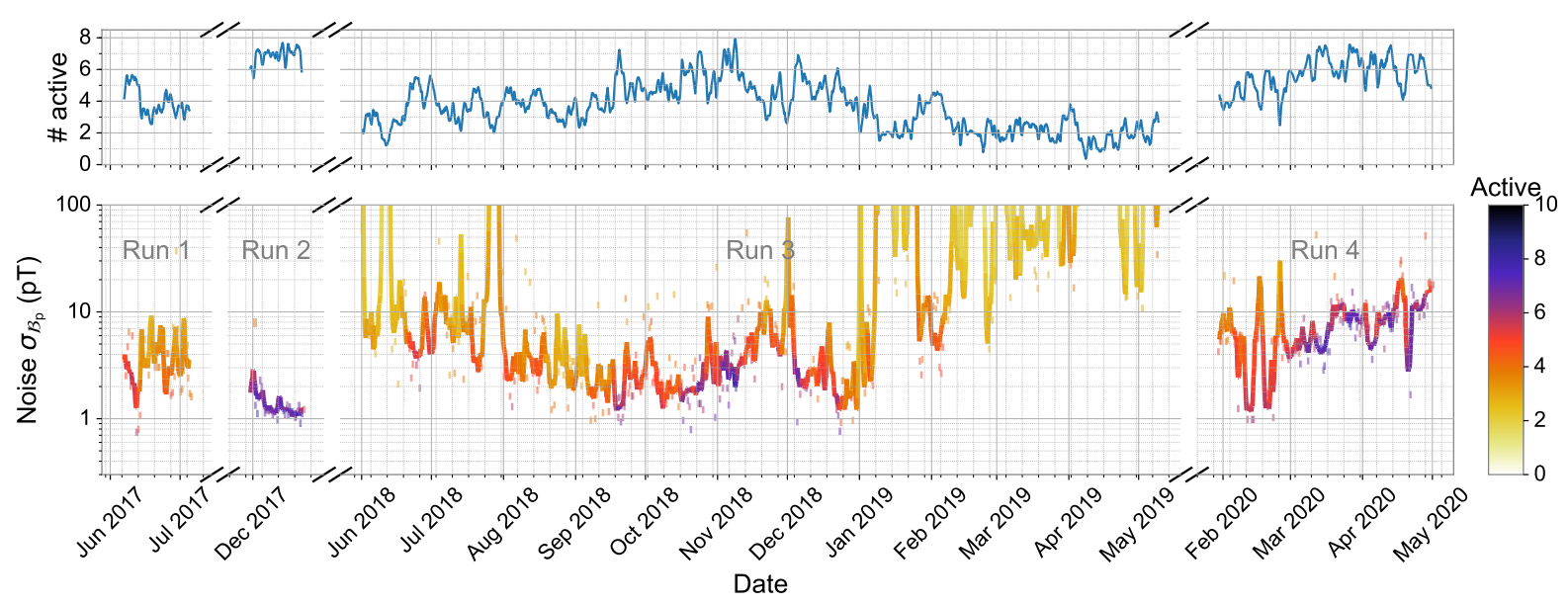

Figure 5. Summary of the GNOME performance during the four Science Runs from 2017 to 2020. The raw magnetometer data are averaged for $20 \mathrm{~s}$ and their standard deviation is calculated over a minimum of one and a maximum of two hours segments depending on the availability of continuous data segments. For each binned point, the combined network noise considering the worse case domain-wall crossing direction is evaluated as defined in Ref. [29]. (a) One-day rolling average of the number of active sensors. (b) Multi-colored solid line represents the one-day rolling average of the combined network noise and the multi-colored dashes show the noise of the individual sampled segments. The data are preprocessed with the same filters used for the analysis. The number of magnetometers active is indicated by the color of the line and dashes.

Table I. Characteristics of the magnetometers active during Science Run 2 (see, e.g., Fig. 5). The station name, location in longitude and latitude, orientation of the sensitive axis, type of magnetometer (NMOR [47, 48], rf-driven [24], or SERF [49]), and probed transition are listed. The bandwidth indicates the measured $-3 \mathrm{~dB}$ point of the magnetometers' frequency response to oscillating magnetic fields. The calibration error takes into account potential temporal variation of the magnetometers' calibration over the course of Science Run 2, and is estimated based on auxiliary measurements. The rightmost column lists the estimated ratio between the effective proton spin polarization and the Landé $g$-factor for the magnetometer, $\sigma_{p} / g$, which depends on the atomic species and the magnetometry scheme as described in Appendix B. The $\sigma_{p} / g$ value is used to relate the measured magnetic field to the signal expected from the interaction of an ALP field with proton spins. The indicated uncertainty describes the range of values from different theoretical calculations [28].

\begin{tabular}{|c|c|c|c|c|c|c|c|c|c|}
\hline \multirow[b]{2}{*}{ Station } & \multicolumn{2}{|c|}{ Location } & \multicolumn{2}{|c|}{ Orientation } & \multirow[b]{2}{*}{ Type } & \multirow[b]{2}{*}{ Probed transition } & \multirow[b]{2}{*}{ Bandwidth } & \multirow[b]{2}{*}{ Cal. Error } & \multirow[b]{2}{*}{$\sigma_{p} / g$} \\
\hline & Longitude & Latitude & $\mathrm{Az}$ & Alt & & & & & \\
\hline Beijing & $116.1868^{\circ} \mathrm{E}$ & $40.2457^{\circ} \mathrm{N}$ & $+251^{\circ}$ & $0^{\circ}$ & NMOR & ${ }^{133} \mathrm{Cs} \mathrm{D} 2 \quad \mathrm{~F}=4$ & $115 \mathrm{~Hz}$ & $20 \%$ & $-0.39_{-0.00}^{+0.19}$ \\
\hline Berkeley & $122.2570^{\circ} \mathrm{W}$ & $37.8723^{\circ} \mathrm{N}$ & $0^{\circ}$ & $+90^{\circ}$ & NMOR & ${ }^{133} \mathrm{Cs} \mathrm{D} 2 \quad \mathrm{~F}=4$ & $7 \mathrm{~Hz}$ & $40 \%$ & $-0.39_{-0.00}^{+0.19}$ \\
\hline Daejeon & $127.3987^{\circ} \mathrm{E}$ & $36.3909^{\circ} \mathrm{N}$ & $0^{\circ}$ & $+90^{\circ}$ & NMOR & ${ }^{133}$ Cs D2 $F=4$ & $10 \mathrm{~Hz}$ & $20 \%$ & $-0.39_{-0.00}^{+0.19}$ \\
\hline Fribourg & $7.1581^{\circ} \mathrm{E}$ & $46.7930^{\circ} \mathrm{N}$ & $+190^{\circ}$ & $0^{\circ}$ & rf-driven & ${ }^{133} \mathrm{Cs} \mathrm{D} 1 \quad \mathrm{~F}=4$ & $94 \mathrm{~Hz}$ & $5 \%$ & $-0.39_{-0.00}^{+0.19}$ \\
\hline Hayward & $122.0539^{\circ} \mathrm{W}$ & $37.6564^{\circ} \mathrm{N}$ & $0^{\circ}$ & $-90^{\circ}$ & NMOR & ${ }^{85} \mathrm{Rb} \mathrm{D} 2 \quad \mathrm{~F}=3$ & $37 \mathrm{~Hz}$ & $5 \%$ & $-0.36_{-0.00}^{+0.05}$ \\
\hline Hefei & $117.2526^{\circ} \mathrm{E}$ & $31.8429^{\circ} \mathrm{N}$ & $+90^{\circ}$ & $0^{\circ}$ & SERF & ${ }^{85} \mathrm{Rb} \&{ }^{87} \mathrm{Rb} \mathrm{D} 1$ & $127 \mathrm{~Hz}$ & $5 \%$ & $-0.38_{-0.00}^{+0.05}$ \\
\hline Krakow & $19.9048^{\circ} \mathrm{E}$ & $50.0289^{\circ} \mathrm{N}$ & $+45^{\circ}$ & $0^{\circ}$ & NMOR & ${ }^{87} \mathrm{Rb} \mathrm{D} 1 \quad \mathrm{~F}=2$ & $3 \mathrm{~Hz}$ & $20 \%$ & $0.50_{-0.11}^{+0.00}$ \\
\hline Lewisburg & $76.8825^{\circ} \mathrm{W}$ & $40.9557^{\circ} \mathrm{N}$ & $0^{\circ}$ & $+90^{\circ}$ & SERF & ${ }^{87} \mathrm{Rb} \mathrm{D} 2$ & $200 \mathrm{~Hz}$ & $10 \%$ & $0.70_{-0.15}^{+0.00}$ \\
\hline Mainz & $8.2354^{\circ} \mathrm{E}$ & $49.9915^{\circ} \mathrm{N}$ & $0^{\circ}$ & $-90^{\circ}$ & NMOR & ${ }^{87} \mathrm{Rb} \mathrm{D} 2 \quad \mathrm{~F}=2$ & $99 \mathrm{~Hz}$ & $2 \%$ & $0.50_{-0.11}^{+0.00}$ \\
\hline
\end{tabular}

529 signal and noise in the same way.

530 Second, in the velocity-scanning stage, the data from 531 the individual magnetometers are time-shifted according 532 to different relative velocities between Earth and the ALP ${ }_{533}$ domain walls. In order to sample $97.5 \%$ of the velocity ${ }_{534}$ probability distribution, a scan of the speeds from 53.7
535 to $770 \mathrm{~km} / \mathrm{s}$ with directions covering the full $4 \pi$ solid 536 angle is chosen. Note that this distribution considers just 537 the observable perpendicular component of the relative 538 domain-wall velocity and neglects the orbital motion of 539 the Earth around the Sun. For low relative velocities, 540 both the time between signals at different magnetometers 
${ }_{541}$ as well as the signal duration diverge. So the velocity 599 false-positive probability. The false-positive analysis is ${ }_{542}$ range is determined by the chosen $97.5 \%$ coverage and 600 performed on the background data. The true-positive ${ }_{543}$ the maximum relative speed of domain walls travelling 601 analysis is performed on test data consisting of back544 at the galactic escape speed.

602 ground data with randomly inserted domain wall signals ${ }_{545}$ The corresponding time-shifted data along with their 603 as we describe below.

${ }_{546}$ local standard deviation estimate are fetched from each ${ }_{604}$ A single signal pattern may appear as multiple po${ }_{547}$ magnetometer's rolling averaged full-rate data at a rate 605 tential events in the analysis, whereas we are seeking ${ }_{548}$ of 0.1 samples/s. This reduces the amount of data to 606 to characterize a single underlying domain-wall crossing 549 process, while keeping the full timing resolution.

607 event. For example, a signal consistent with a domain${ }_{550}$ The step size used in the speed scan is chosen so that 608 wall crossing lasting for multiple sampling periods would ${ }_{551}$ a single step in speed corresponds to time-shift differ- 609 appear as multiple potential events in a single time552 ences of less than the down-sampled sampling period. 610 shifted data set. Furthermore, even if such a signal lasts ${ }_{553}$ For each speed, a lattice of directions covering the full 611 for only a single sampling period, corresponding potential ${ }_{554} 4 \pi$ solid angle is constructed. The angular difference be- 612 events appear in different time-shifted data sets. Since 555 tween adjacent directions is informed by sampling rate 613 it is assumed that domain-wall crossings occur rarely, ${ }_{556}$ and speed [29] such that, as for the speed scan, a single 614 such clusters of potential events are classified as a sin${ }_{557}$ step in direction results in time-shift differences of less 615 gle "event." In order to reduce double-counting of these ${ }_{558}$ than the down-sampled sampling period. With the set- 616 events, conditions are imposed. If potential events pass559 tings used, the velocity-scanning lattice consists of 1661 617 ing the thresholds occur at the same time in different 560 points. This number scales with the down-sampled sam- 618 time-shifted data sets or are contiguous in time, the po${ }_{561}$ pling rate cubed. 619 tential event with the greatest magnitude-to-uncertainty ${ }_{562}$ After time-shifting, the pulses produced by a domain- ${ }^{620}$ ratio is classified as the corresponding single event.

${ }_{563}$ wall crossing appear simultaneously as if all the magne- ${ }_{621}$ The true-positive analysis studies the detection prob564 tometers were placed at the Earth's center. This process ${ }_{622}$ ability as a function of the thresholds. Multiple test ${ }_{565}$ results in a time-shifted data set for each lattice veloc- ${ }_{623}$ data sets are created featuring domain-wall-signal pat${ }_{566}$ ity on which for each time point a $\chi^{2}$-minimization is 624 terns with random parameters by inserting Lorentzian${ }_{567}$ performed to estimate the domain-wall parameters. An ${ }_{625}$ shaped pulses into the background data of the different ${ }_{568}$ ALP domain-wall-crossing direction and magnitude, $\mathcal{B}_{\mathrm{p}}$, ${ }_{226}$ GNOME magnetometers. The domain-crossing events ${ }_{569}$ with the corresponding $p$-value quantifying the agree- ${ }_{627}$ have magnitudes of $\mathcal{B}_{\mathrm{p}}$ randomly selected between $0.1 \mathrm{pT}$ 570 ment is obtained. The $p$-value is evaluated as the prob- ${ }_{628}$ and $10^{4} \mathrm{pT}$ and durations randomly selected between 571 ability of obtaining the given $\chi^{2}$ value or higher from $6290.01 \mathrm{~s}$ and $10^{3} \mathrm{~s}$. The distributions of the these ran572 the $\chi^{2}$-minimization. The $p$-value is calculated using the ${ }_{630}$ domized parameters are chosen to be flat on a logarith573 quadrature sum of the standard deviation of the data and ${ }_{631}$ mic scale. Additionally, the signals are inserted at ran574 the uncertainty due to drifts of the calibration factors. ${ }_{632}$ dom times with random directions. In order to simulate 575 All data points in every time-shifted data set are consid- 633 calibration error effects, the pulse amplitudes inserted 576 ered potential events, characterized by time, $p$-value as 634 in each magnetometer are weighted by a random factor ${ }_{577}$ well as direction and magnitude $\mathcal{B}_{\mathrm{p}}$ with their associated ${ }_{635}$ whose range is given in Table I. The crossing velocity is 578 uncertainties. The magnitude-to-uncertainty ratio of an 636 also randomized within the range covered by the veloc${ }_{579}$ event $\zeta$ is the ratio between this $\mathcal{B}_{\mathrm{p}}$ and its associated ${ }_{637}$ ity lattice. For each inserted domain-wall-crossing event, 580 uncertainty. ${ }_{638}$ the $p$-value, the normalized angular difference, and the ${ }_{581}$ Third, in the post-selection stage, two tests are carried 639 magnitude-to-uncertainty ratio are computed.

582 out to check if a potential event is consistent with an ${ }_{583}$ ALP domain-wall crossing. The domain-wall model test ${ }_{584}$ evaluates if the observed signal amplitudes are consistent 585 with the expected pattern of a domain-wall crossing from 586 any possible direction. It is quantified by the aforemen${ }_{587}$ tioned $p$-value. The directional-consistency test is based 588 on the angular difference between the estimated domain589 wall crossing direction and the direction of the velocity 590 corresponding to the particular time-shifted data set be591 ing analyzed. In a real domain-wall crossing event, these 592 two directions should be aligned.

Figure $6 \mathrm{a}$ shows the detection probability as a func${ }_{641}$ tion of the threshold on the lower-limit of the $p$-value 642 and the threshold on the upper-limit of the normalized 643 angular difference. We restrict the analysis in Fig. 6a 644 to events inserted with a magnitude-to-uncertainty ra645 tio between 5 and 10. This enables reliable determina646 tion of the true-positive detection probability without ${ }_{647}$ significant contamination by false positive events since ${ }_{648}$ the background event probability above $\zeta=5$ is below ${ }_{649} 0.01 \%$ in a 10 s sampling interval. Since the detection ${ }_{650}$ probability increases with the signal magnitude, we fo${ }_{593}$ To evaluate the consistency of a potential event with ${ }_{651}$ cus on the events below $\zeta=10$. The detection proba${ }_{594}$ a domain-wall crossing, we impose thresholds on the $p$ - 652 bility is then the number of detected events divided by ${ }_{595}$ value and the angular difference normalized with respect 653 the number of inserted events. The black line marks the 596 to the angular spacing of the lattice of velocity points for 654 numerically evaluated boundary of the area guaranteeing ${ }_{597}$ that speed. The thresholds are chosen to guarantee a de- 655 at least $97.5 \%$ detection. All points along this black line ${ }_{598}$ tection probability of $97.5 \%$ with the minimum possible 656 will yield the desired detection probability, so the partic- 

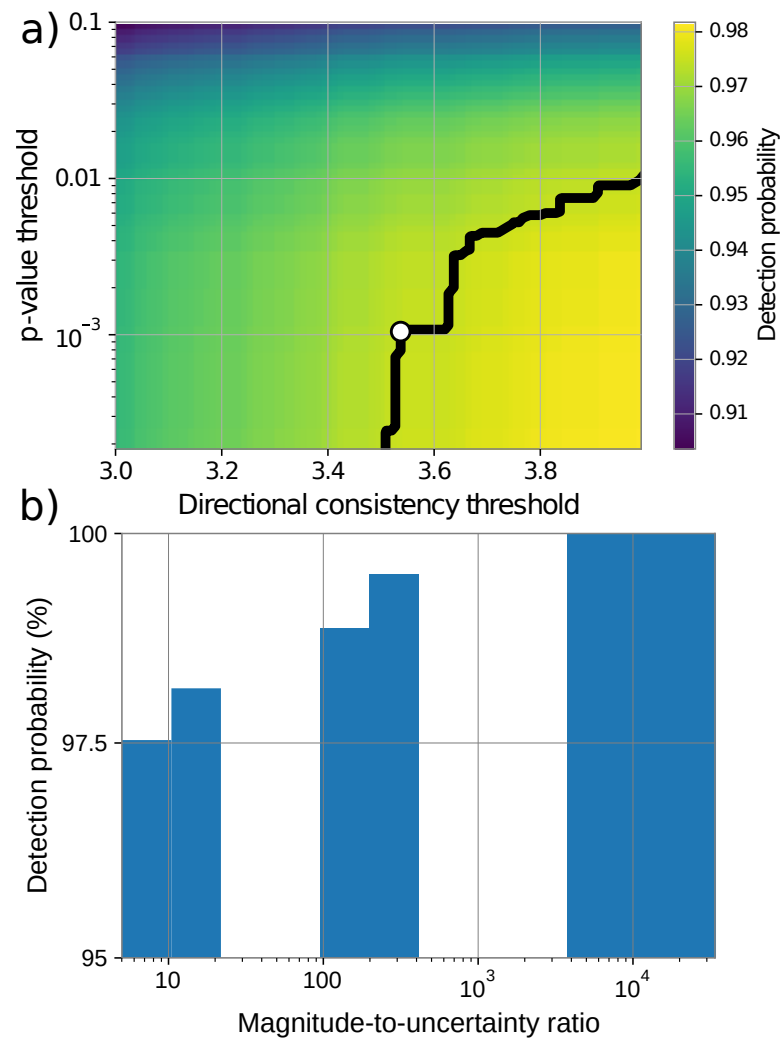

Figure 6. Summary of the true-positive analysis results. (a) shows the probability of detecting a domain-wall-crossing event with randomized parameters (as discussed in the text) as a function of $p$-value and directional-consistency thresholds. The inserted events have a magnitude-to-uncertainty ratio between 5 and 10 . The black line indicates the combination of parameters corresponding to a $97.5 \%$ detection probability. The white dot indicates the particular thresholds chosen for the analysis. (b) Shows the mean detection probability reached for different magnitude-to-uncertainty ratios for the chosen thresholds.

${ }_{657}$ ular choice is made to minimize the number of candidate ${ }_{658}$ events when applying the search algorithm to background 659 data. These values are 0.001 for the $p$-value threshold 660 and 3.5 for the directional-consistency threshold (repre${ }_{661}$ sented as a white dot in Fig. 6a). Figure 6b shows that ${ }_{662}$ the detection probability is greater than $97.5 \%$ for events ${ }_{663}$ featuring a magnitude-to-uncertainty ratio above 5 and ${ }_{664}$ guarantees $\epsilon \geq 95 \%$.

${ }_{665}$ Since the noise has a nonzero probability of mimicking 666 the signal pattern expected from an ALP domain-wall ${ }_{667}$ crossing well enough to pass the $p$-value and directional ${ }_{668}$ consistency tests, we perform a false-positive study on 669 background data. The analysis algorithm is applied to ${ }_{670} \mathrm{~T}_{b}=10.7$ years of time-shuffled data in order to establish 671 the rate of events expected solely from background. Be672 cause of the larger amount of background data analyzed, 673 lower rates and larger magnitude-to-uncertainty ratios 674 are accessible as compared to the search data. Based on ${ }_{723}$ The ALP parameter space is constrained by imposing

675 the false positive study, the probability of finding one or 724 that $r \geq R_{90 \%}$. The experimental constraint on the
676 more events in the search data above $\zeta$, is [50],

$$
P(\geq 1 \text { above } \zeta)=1-\exp \left(-\frac{T}{T_{b}}\left[1+n_{b}(\zeta)\right]\right),
$$

677 where $T=23$ days is the duration of Science Run 2 678 and $n_{b}(\zeta)$ is the number of candidate events found in the 679 background data above $\zeta$. The significance is then de680 fined as, $\mathrm{S}=-\sqrt{2} \operatorname{erf}^{-1}[1-2(1-P)]$, where $\operatorname{erf}^{-1}$ is the 681 inverse error function. The significance is given in units 682 of the Gaussian standard deviation which corresponds to 683 a one-sided probability of $P$.

${ }_{684}$ After characterizing the background for Science Run 2, 685 the search data are analyzed. The results are represented 686 as a solid green line in Fig. 2. For $\zeta>6$ only a few ${ }_{687}$ events were found. The event with largest magnitude688 to-uncertainty ratio, $\zeta_{\max }$ was measured at 12.6 followed 689 by additional events at 6.2 and 5.6. From Eq. (10), the 690 significance associated with finding one or more events ${ }_{691}$ produced by the background featuring at least $\zeta_{\max }$ is ${ }_{692}$ lower than one sigma. This null result defines the sensi${ }_{693}$ tivity of the search and is used to set constraints on the ${ }_{694}$ parameter space describing ALP domain walls.

${ }_{695}$ The observable rate of domain-wall crossings depends 696 on how long GNOME was sensitive to different signal du697 rations and magnitudes. For the evaluation of this effec698 tive time, the raw data of each magnetometer are divided 699 into continuous segments between one to two hours dura700 tion depending on the availability of the data. The pre701 processing steps are applied to each segment. Then the 702 data are binned by taking the average in 20 s intervals. 703 To estimate the noise in each magnetometer, the stan704 dard deviation in each binned segment is calculated to 705 define the covariance matrix $\Sigma_{s}$. The domain-wall mag706 nitude, crossing with the worse case direction $\hat{\boldsymbol{m}}$, needed 707 to produce $\zeta=1$ is calculated as in Ref. [29],

$$
\mathcal{B}_{\mathrm{p}}^{\prime}(\Delta t)=\sqrt{\hat{\boldsymbol{m}}\left(D_{\Delta t}^{T} \Sigma_{s}^{-1} D_{\Delta t}\right)^{-1} \hat{\boldsymbol{m}}},
$$

708 for each bin. The matrix $D_{\Delta t}$ contains the sensitivity 709 axes of the magnetometers, the factor $\sigma_{p} / g$, and the ef710 fects of the preprocessing as a function of the signal dura711 tion as described in Ref. [29]. Such prepocessing effects 712 rely on a Lorentzian-shaped signal and give rise to the 713 characteristic shape of Fig. 3. The effective time, $\tilde{T}$, is 714 defined as the amount of time the network can measure 715 a domain wall with duration $\Delta t$ and magnitude $\mathcal{B}_{\mathrm{p}}^{\prime}$ pro${ }_{716}$ ducing $\zeta \geq 1$. Monte Carlo simulations analyzing seg717 ments with inserted domain-wall encounters on raw data ${ }_{718}$ show a good agreement with the sensitivity estimation in ${ }_{719}$ Eq. (11).

Assuming that the domain-wall encounters follow Pois721 son statistics, a bound on the observable rate of events ${ }^{722}$ above $\zeta_{\max }$ with $90 \%$ confidence is set as [40],

$$
R_{90 \%}=\frac{-\log (0.1)}{\epsilon \tilde{T}\left(\Delta t, \mathcal{B}_{\mathrm{p}}^{\prime}\right)} .
$$


725 coupling constant is written as (see Eq. (A.13) in Ap726 pendix A),

$$
f_{\text {int }} \leq \frac{\hbar}{\xi} \sqrt{\frac{\bar{v} \rho_{\mathrm{DW} \epsilon}}{8 m_{a} \log (0.1)} \tilde{T}\left(\Delta t, \mathcal{B}_{\mathrm{p}}^{\prime}\right)}
$$

${ }_{727}$ The signal duration can be written in terms of the mass 728 of the hypothetical ALP particle and the specific domain729 wall crossing speed, $\Delta t=\frac{2 \sqrt{2} \hbar}{\bar{v} m_{a} c}$. When calculating the 730 constraints on $f_{\text {int }}$, we fix the domain-wall crossing speed 731 to the typical relative speed from the Standard Halo ${ }_{732}$ Model, $\bar{v}=300 \mathrm{~km} \mathrm{~s}^{-1}$ [31]. In contrast to the sig733 nal duration, the pseudo-magnetic field signal depends ${ }_{734}$ on all parameters of the ALPs, the mass and the ra735 tio between the coupling and symmetry breaking con736 stants, $\mathcal{B}_{\mathrm{p}}^{\prime}=\frac{4 m_{a} c^{2} \xi}{\mu_{B} \zeta}$. Figure 4 is given by Eq. (13) taking ${ }_{737} \zeta=12.6$. The shape of the constrained space is given by ${ }_{738}$ the fact that $\tilde{T}$ varies depending on the target $m_{a}$ and $\xi$. 739 A detailed derivation of Eq. (13) is given in Appendix A.

\section{ACKNOWLEDGMENTS}

741 The authors are grateful to Chris Pankow, Joshua ${ }_{742}$ R. Smith, Jocelyn Read, Menachem Givon, Ron Fol-
743 man, Wojciech Gawlik, Kathryn Grimm, Grzegorz ${ }_{744}$ Lukasiewicz, Peter Fierlinger, Volkmar Schultze, Tilman 745 Sander-Thömes, and Holger Müller for insightful discus746 sions.

This work was supported by the U.S. National Sci748 ence Foundation under grants PHY-1707875 and PHY749 1707803, the Swiss National Science Foundation under 750 grant No. 200021 172686, the German research founda751 tion under grant no. 439720477, the European Research ${ }_{752}$ Council under the European Union's Horizon 2020 Re753 search and Innovative Program under Grant agreement ${ }_{754}$ No. 695405, the Cluster of Excellence PRISMA+, DFG 755 Reinhart Koselleck Project, Simons Foundation, a Fun756 damental Physics Innovation Award from the Gordon 757 and Betty Moore Foundation, Heising-Simons Founda758 tion, the National Science Centre of Poland within the 759 OPUS program (Project No. 2015/19/B/ST2/02129), 760 and IBS-R017-D1-2021-a00 of the Republic of Korea.
792 [12] A. Vilenkin, Cosmic strings and domain walls, Phys. Rep. 824

121, 263 (1985). Evidence, candidates and constraints, Phys. Rep. 405, 79 279 (2005).

794

2] J. L. Feng, Dark matter candidates from particle physics 796 and methods of detection, Annu. Rev. Astron. Astrophys. 797 48, 495 (2010).

[3] P. Gorenstein and W. Tucker, Astronomical signatures 799 of dark matter, Adv. High Energy Phys. 2014, 878203800 (2014).

[4] M. Safronova, D. Budker, D. DeMille, D. F. Jackson Kim- ${ }^{802}$ ball, A. Derevianko, and C. W. Clark, Search for new 803 physics with atoms and molecules, Rev. Mod. Phys. 90, 804 025008 (2018).

[5] J. Preskill, M. B. Wise, and F. Wilczek, Cosmology of 806 the invisible axion, Phys. Lett. B 120, 127 (1983). ${ }_{807}$

[6] L. F. Abbott and P. Sikivie, A cosmological bound on 808 the invisible axion, Phys. Lett. B 120, 133 (1983).

] M. Dine and W. Fischler, The not-so-harmless axion, 810 Phys. Lett. B 120, 137 (1983).

[8] P. W. Graham, I. G. Irastorza, S. K. Lamoreaux, A. Lind- 812 ner, and K. A. van Bibber, Experimental searches for the 813 axion and axion-like particles, Annu. Rev. Nucl. Part. 8 Sci. 65, 485 (2015).

[9] P. W. Graham, D. E. Kaplan, and S. Rajendran, Cos- 816 mological relaxation of the electroweak scale, Phys. Rev. ${ }^{817}$ Lett. 115, 221801 (2015).

R. T. Co, L. J. Hall, and K. Harigaya, Predictions 81 for axion couplings from alp cogenesis, arXiv:2006.04809 820 (2020).
13] M. Pospelov, S. Pustelny, M. P. Ledbetter, D. F. Jackson Kimball, W. Gawlik, and D. Budker, Detecting domain walls of axionlike models using terrestrial experiments, Phys. Rev. Lett. 110, 021803 (2013).

[14] M. Kawasaki, K. Saikawa, and T. Sekiguchi, Axion dark matter from topological defects, Phys. Rev. D 91, 065014 (2015).

[15] D. J. Kaup, Klein-gordon geon, Phys. Rev. 172, 1331 (1968).

[16] E. Braaten, A. Mohapatra, and H. Zhang, Dense axion stars, Phys. Rev. Lett. 117, 121801 (2016).

[17] D. Jackson Kimball, D. Budker, J. Eby, M. Pospelov, S. Pustelny, T. Scholtes, Y. Stadnik, A. Weis, and A. Wickenbrock, Searching for axion stars and q-balls with a terrestrial magnetometer network, Phys. Rev. D 97, 043002 (2018).

[18] A. Derevianko and M. Pospelov, Hunting for topological dark matter with atomic clocks, Nat. Phys. 10, 933 (2014).

[19] S. Coleman, Q-balls, Nucl. Phys. B 262, 263 (1985).

[20] A. Kusenko and P. J. Steinhardt, Q-ball candidates for self-interacting dark matter, Phys. Rev. Lett. 87, 141301 (2001).

[21] S. Pustelny, D. F. Jackson Kimball, C. Pankow, M. P. Ledbetter, P. Wlodarczyk, P. Wcislo, M. Pospelov, J. R. Smith, J. Read, W. Gawlik, and D. Budker, The global network of optical magnetometers for exotic physics (gnome): A novel scheme to search for physics beyond the standard model, Ann. Phys. (Berl.) 525, 659 (2013).

2 B. M. Roberts, G. Blewitt, C. Dailey, M. Murphy, M. Pospelov, A. Rollings, J. Sherman, W. Williams, and 
A. Derevianko, Gps as a dark matter detector: Orders- 889 of-magnitude improvement on couplings of clumpy dark 890 matter to atomic clocks, arXiv:1704.06844, Nat. Com- 891 mun. (2017).

23] J. Bovy and S. Tremaine, On the local dark matter den- 893 sity, Astrophys. J. 756, 89 (2012).

24] S. Afach, D. Budker, G. DeCamp, V. Dumont, Z. D. 895 Grujić, H. Guo, D. Jackson Kimball, T. Kornack, 896 V. Lebedev, W. Li, et al., Characterization of the global ${ }^{897}$ network of optical magnetometers to search for exotic 898 physics (gnome), Phys. Dark Universe 22, 162 (2018). $\quad 899$

25] P. Włodarczyk, S. Pustelny, D. Budker, and M. Lipiński, 900 Multi-channel data acquisition system with absolute time 901 synchronization, Nucl. Instr. Meth. Phys. Res. A 763, 902 150 (2014).

V. V. Yashchuk, S.-K. Lee, and E. Paperno, Magnetic 904 shielding, in Optical Magnetometry, edited by D. Budker 905 and D. F. Jackson Kimball (Cambridge University Press, 906 2013) Chap. 12.

27] D. F. Jackson Kimball, J. Dudley, Y. Li, S. Thulasi, 908 S. Pustelny, D. Budker, and M. Zolotorev, Magnetic 909 shielding and exotic spin-dependent interactions, Phys. 910 Rev. D 94, 082005 (2016).

28] D. F. Jackson Kimball, Nuclear spin content and con- 912 straints on exotic spin-dependent couplings, New J. Phys. 913 17, 073008 (2015).

[29] H. Masia-Roig, J. A. Smiga, D. Budker, V. Dumont, 915 Z. Grujic, D. Kim, D. F. Jackson Kimball, V. Lebedev, 916 M. Monroy, S. Pustelny, T. Scholtes, P. C. Segura, Y. K. 917 Semertzidis, Y. C. Shin, J. E. Stalnaker, I. Sulai, A. Weis, 918 and A. Wickenbrock, Analysis method for detecting topo- 919 logical defect dark matter with a global magnetometer 920 network, Phys. Dark Universe 28, 100494 (2020).

30] C. Dailey, C. Bradley, D. F. Jackson Kimball, I. Sulai, ${ }_{922}$ S. Pustelny, A. Wickenbrock, and A. Derevianko, Quan- ${ }_{923}$ tum sensor networks as exotic field telescopes for multi- 924 messenger astronomy, Nat. Astron. (2020).

31] B. M. Roberts, G. Blewitt, C. Dailey, M. Murphy, 926 M. Pospelov, A. Rollings, J. Sherman, W. Williams, and 927 A. Derevianko, Search for domain wall dark matter with 928 atomic clocks on board global positioning system satel- 929 lites, Nat. Commun. 8, 1 (2017).

22] P. Wcisło, P. Morzyński, M. Bober, A. Cygan, D. Lisak, 931 R. Ciuryło, and M. Zawada, Experimental constraint on 932 dark matter detection with optical atomic clocks, Nat. 933 Astron. 1, 1 (2016).

33] B. Roberts, P. Delva, A. Al-Masoudi, A. Amy-Klein, 935 C. Baerentsen, C. Baynham, E. Benkler, S. Bilicki, 936 W. Bowden, E. Cantin, et al., Search for transient vari- ${ }^{937}$ ations of the fine structure constant and dark matter ${ }_{938}$ using fiber-linked optical atomic clocks, arXiv preprint 939 arXiv:1907.02661 (2019). Y. V. Stadnik and V. V. Flambaum, Searching for dark 94 matter and variation of fundamental constants with laser 942 and maser interferometry, Phys. Rev. Lett. 114, 161301943 (2015).

35] Y. V. Stadnik and V. V. Flambaum, Enhanced effects of 945 variation of the fundamental constants in laser interfer- 946 ometers and application to dark-matter detection, Phys. ${ }_{947}$ Rev. A 93, 063630 (2016).

${ }_{888}$ [37] A. Arvanitaki, S. Dimopoulos, and K. Van Tilburg,
Sound of dark matter: Searching for light scalars with resonant-mass detectors, Phys. Rev. Lett. 116, 031102 (2016).

[38] R. L. McNally and T. Zelevinsky, Constraining domain wall dark matter with a network of superconducting gravimeters and ligo, Eur. Phys. J. D 74, 1 (2020).

[39] B. P. Abbott, R. Abbott, T. D. Abbott, M. R. Abernathy, F. Acernese, K. Ackley, C. Adams, T. Adams, P. Addesso, R. X. Adhikari, V. B. Adya, C. Affeldt, M. Agathos, K. Agatsuma, N. Aggarwal, O. D. Aguiar, L. Aiello, A. Ain, P. Ajith, B. Allen, A. Allocca, P. A. Altin, S. B. Anderson, W. G. Anderson, K. Arai, and Araya (LIGO Scientific Collaboration and Virgo Collaboration), Gw150914: First results from the search for binary black hole coalescence with advanced ligo, Phys. Rev. D 93, 122003 (2016).

[40] P. R. Brady, J. D. E. Creighton, and A. G. Wiseman, Upper limits on gravitational-wave signals based on loudest events, Class. Quantum Grav. 21, S1775 (2004).

[41] N. F. Ramsey, The tensor force between two protons at long range, Physica A: Statistical Mechanics and its Applications 96, 285 (1979).

[42] W. DeRocco, P. W. Graham, and S. Rajendran, Exploring the robustness of stellar cooling constraints on light particles, Phys. Rev. D 102, 075015 (2020).

[43] N. Bar, K. Blum, and G. D'amico, Is there a supernova bound on axions?, Phys. Rev. D 101, 123025 (2020).

[44] T. W. Kornack and M. V. Romalis, Dynamics of two overlapping spin ensembles interacting by spin exchange, Phys. Rev. Lett. 89, 253002 (2002).

[45] T. W. Kornack, R. K. Ghosh, and M. V. Romalis, Nuclear spin gyroscope based on an atomic comagnetometer, Phys. Rev. Lett. 95, 230801 (2005).

[46] A. Banerjee, D. Budker, J. Eby, H. Kim, and G. Perez, Relaxion stars and their detection via atomic physics, Commun. Phys. 2 (2020).

[47] D. Budker, D. Kimball, V. Yashchuk, and M. Zolotorev, Nonlinear magneto-optical rotation with frequencymodulated light, Phys. Rev. A 65, 055403 (2002).

[48] W. Gawlik, L. Krzemień, S. Pustelny, D. Sangla, J. Zachorowski, M. Graf, A. Sushkov, and D. Budker, Nonlinear magneto-optical rotation with amplitude modulated light, Appl. Phys. Lett. 88, 131108 (2006).

[49] J. Allred, R. Lyman, T. Kornack, and M. V. Romalis, High-sensitivity atomic magnetometer unaffected by spin-exchange relaxation, Phys. Rev. Lett. 89, 130801 (2002).

[50] S. A. Usman, A. H. Nitz, I. W. Harry, C. M. Biwer, D. A. Brown, M. Cabero, C. D. Capano, T. D. Canton, T. Dent, S. Fairhurst, M. S. Kehl, D. Keppel, B. Krishnan, A. Lenon, A. Lundgren, A. B. Nielsen, L. P. Pekowsky, H. P. Pfeiffer, P. R. Saulson, M. West, and J. L. Willis, The PyCBC search for gravitational waves from compact binary coalescence, Class. Quantum Grav. 33, 215004 (2016).

[51] R. D. Peccei and H. R. Quinn, Cp conservation in the presence of instantons, Phys. Rev. Lett. 38, 1440 (1977).

[52] I. Kominis, T. Kornack, J. Allred, and M. V. Romalis, A subfemtotesla multichannel atomic magnetometer, Nature 422, 596 (2003).

[53] M. Ledbetter, I. Savukov, V. Acosta, D. Budker, and M. Romalis, Spin-exchange-relaxation-free magnetometry with cs vapor, Phys. Rev. A 77, 033408 (2008). 
952 [54] T. Schmidt, Über die magnetischen momente der atom${ }_{953}$ kerne, Z. Phys. 106, 358 (1937).

${ }_{954}^{\text {[55] J. Engel and P. Vogel, Spin-dependent cross sections of } 1000}$ 955 weakly interacting massive particles on nuclei, Phys. Rev.

956 D 40, 3132 (1989).

${ }_{957}$ [56] V. Flambaum and A. Tedesco, Dependence of nuclear 958 magnetic moments on quark masses and limits on tempo959 ral variation of fundamental constants from atomic clock 960 experiments, Phys. Rev. C 73, 055501 (2006).

961 [57] J. Engel, M. Ressell, I. Towner, and W. Ormand, RePhys. Rev. C 52, 2216 (1995)

58] P. Toivanen, M. Kortelainen, J. Suhonen, and J. Toivanen, Large-scale shell-model calculations of elastic and inelastic scattering rates of lightest supersymmetric particles (lsp) on i 127, xe 129, xe

5] F. Iachello, L. M. Krauss, and G. Maino, Spin-dependent 1 scattering of weakly interacting massive particles in heavy nuclei, Physics Letters B 254, 220 (1991).

[60] L. W. Anderson, F. M. Pipkin, and J. C. Baird Jr, N-14 - N-15 Hyperfine Anomaly, Phys, Rev. 116, 87 (1959).

61] W. Happer and H. Tang, Spin-exchange shift and narrowing of magnetic resonance lines in optically pumped alkali vapors, Phys. Rev. Lett. 31, 273 (1973).

$62]$ I. Savukov and M. Romalis, Effects of spin-exchange collisions in a high-density alkali-metal vapor in low magnetic fields, Phys. Rev. A 71, 023405 (2005).

63] D. Budker, D. F. Kimball, and D. P. DeMille, Atomic physics: an exploration through problems and solutions (Oxford University Press, USA, 2008).

A. Young, and W. Happer, Theory of spin-exchange optical pumping of 3 he and 129 xe, Phys. Rev. A 58, 1412 (1998).
The axion field is obtained by reparameterizing the 1000 complex field $\phi$ in Eq. (A.1) in terms of the real field $S$ (Higgs) and $a$ (axion),

$$
\phi=\frac{S}{\sqrt{2}} \exp \left(i a / S_{0}\right) .
$$

1002 The second term in Eq. (A.1) will break the $U(1)$ sym1003 metry of the complex field into a discrete $\mathbb{Z}_{N}$ symmetry, ${ }_{1004} \phi \rightarrow \exp (2 \pi i k / N) \phi$ for integer $k$. This corresponds to 1005 the axion shift-symmetry, $a \rightarrow a+\frac{2 \pi S_{0}}{N} k$. The Higgs 1006 mode obtains a vacuum expectation value $S \rightarrow S_{0}$, and 1007 the axion field has degenerate vacua or ground energy 1008 states at $a=\frac{\pi S_{0}}{N}(2 k+1)$ for integer $k$. One can define 1009 the symmetry-breaking scale as $f_{\mathrm{SB}}=S_{0} / N$. Reparame1010 terizing the complex scalar field in Eq. (A.1) and setting 1011 the Higgs mode to the vacuum expectation value, the 12 axion Lagrangian is

$$
\mathcal{L}_{a}=\frac{1}{2}\left(\partial_{\mu} a\right)^{2}-2 m_{a}^{2} f_{\mathrm{SB}}^{2} \cos ^{2}\left(\frac{a}{2 f_{\mathrm{SB}}}\right)
$$

1013 where the axion mass is $m_{a}=N S_{0} \sqrt{2 \lambda}$. This can be seen 1014 by matching the second-derivative of the cosine term at 1015 the minima to a scalar mass term.

1016 For simplicity, a static domain wall in the $y z$-plane 1017 separating domains of $-\pi f_{\mathrm{SB}}$ and $+\pi f_{\mathrm{SB}}$ is considered. 1018 Solving the classical field equations, one finds

$$
a(x)=2 f_{\mathrm{SB}} \arcsin \left[\tanh \left(m_{a} x\right)\right] .
$$

1019 The gradient of the field is then

$$
\frac{d a}{d x}(x)=\frac{2 f_{\mathrm{SB}} m_{a}}{\cosh \left(m_{a} x\right)} .
$$

987 Appendix A: Relating measured parameters to ALP ${ }_{1020}$ This has the full width at half maximum, domain-wall parameter space

989 Domain walls form when a field can monotonically vary 990 across vacuum states; two degenerate vacua or possibly 991 the same state if, for example, the field takes values on 992 the 1-sphere. This is the case for ALPs which arise from 993 the angular part of a complex scalar field [51].

${ }_{994}$ The following Lagrangian terms are considered in nat995 ural units ( $\hbar=c=1$ here and throughout this appendix) 996 for a new complex scalar field $\phi,{ }^{4}$

$$
\mathcal{L} \supset\left|\partial_{\mu} \phi\right|^{2}-\frac{\lambda}{S_{0}^{2 N-4}}\left|2^{N / 2} \phi^{N}+S_{0}^{N}\right|^{2},
$$

${ }_{1024}$ Interactions observable by magnetometers involve cou1025 pling between the axion field gradient and the axial1026 vector current of a fermionic field. For a fermion field

$$
\Delta x=\frac{2 \cosh ^{-1}(2)}{m_{a}} \approx \frac{2 \sqrt{2}}{m_{a}} .
$$

1021 Using the domain-wall solution [Eq. (A.3)] and integrat1022 ing the energy density of the domain wall over $x$ yields 1023 the surface tension (energy per unit area) [13],

$$
\sigma_{\mathrm{DW}}=8 m_{a} f_{\mathrm{SB}}^{2} .
$$

997 where $\lambda$ is a unitless constant and $S_{0}$ is a constant with ${ }_{1028}$ 998 units of energy [13].

4 Other references may use a minus sign in front of the $S_{0}^{N}$ term, which results in a similar potential, up to a phase. The end result of Eq. (A.1) is that the axion potential will have a maximum at zero, while the minus-convention will have a vacuum at zero.
${ }_{1027} \psi$, the interaction is

$$
\begin{aligned}
\mathcal{L}_{\mathrm{int}}= & \frac{i\left(\phi \partial_{\mu} \phi^{*}-\partial_{\mu} \phi \phi^{*}\right)}{S_{0} f_{\mathrm{int}}} \bar{\psi} \gamma^{\mu} \gamma^{5} \psi \\
& \stackrel{S \rightarrow S_{0}}{\longrightarrow} \frac{\partial_{\mu} a}{f_{\mathrm{int}}} \bar{\psi} \gamma^{\mu} \gamma^{5} \psi
\end{aligned}
$$

${ }_{031}$ The axial-vector current is related to the spin $\boldsymbol{S}$, so that 1032 the interaction Hamiltonian becomes

$$
H_{\mathrm{int}}=\frac{1}{f_{\mathrm{int}}} \nabla a \cdot \frac{\boldsymbol{S}}{\|S\|},
$$


1033 i.e., for spin- $1 / 2$ particles, $1 /\|S\|=2$.

1078 density $\rho_{\mathrm{DW}}$ and the typical relative speed $\bar{v}$ are assumed ${ }_{1034}$ Optical magnetometers operate by measuring the 1079 according to the observed dark matter energy density and 1035 change in atomic energy levels between two energy states 1080 the galactic rotation velocity, respectively.

1036 with magnetic quantum numbers differing by $\Delta m_{F}$. A ${ }_{1081}$ In order to determine if a set of physical parameters is 1037 leading magnetic field is applied to the atoms, and vari- 1082 observable, the likelihood that no events are found must 1038 ations in the magnetic field along the leading field are 1083 be constrained. This constraint defines the confidence 1039 measured. The spin coupling from Eq. (A.8) can in- 1084 level of the detection. The probability of observing $k$ 1040 duce a similar shift in energy levels to a magnetic field. ${ }_{1085}$ events given that one expects to observe $\mu$ events is given 1041 The maximum energy shift is determined by plugging the 1086 by the Poisson probability mass function, 1042 largest gradient from Eq. (A.4) into Eq. (A.8),

$$
\Delta E=\sum_{i \in \mathrm{e}, \mathrm{p}, \mathrm{n}, \ldots} \frac{2 \eta \sigma_{(i)} \Delta m_{F}}{\left\|S_{(i)}\right\|} \frac{f_{\mathrm{SB}}}{f_{\mathrm{int}}^{(i)}} m_{a}
$$

$$
P(k ; \mu)=\frac{\mu^{k}}{k !} e^{-\mu} .
$$

1087 However, the network also has some detection efficiency ${ }_{1088} \epsilon<1$, so there could be multiple domain-wall-crossing 1043 where $i$ labels the species of fermion, $\sigma_{(i)}=\frac{\left\langle\boldsymbol{S}_{(i)} \cdot \boldsymbol{F}_{(i)}\right\rangle}{\boldsymbol{F}_{(i)}^{2}}{ }_{1089}$ events, but no detection. In particular, the chance of 1044 is the projected spin coupling, $\eta=\cos \theta$ for the an- $1091 \epsilon)^{k}$. For an event rate $r$ and measurement time $T$, the 1045 gle between the axion gradient and sensitive axis $\theta$, and 1092 probability that no events are detected is then $1046 f_{\text {int }}^{(i)}$ is the interaction coupling to particle $i$. In general, 1047 we will combine the $\frac{\sigma_{(i)}}{\left\|S_{(i)}\right\| f_{\text {int }}^{(i)}}$ terms into an effective ra1048 tio $\frac{2 \sigma_{j}}{f_{\text {int }}}$, where $j$ now labels the magnetometer. Com1049 paring this to the energy shift due to a magnetic field, $1050 \Delta E_{B}=g_{F, j} \mu_{B} \Delta m_{F} B_{j}$, one obtains a relationship for a 1051 normalized pseudo-magnetic field,

$$
\sum_{k=0}^{\infty}(1-\epsilon)^{k} \frac{(r T)^{k}}{k !} e^{-r T}=e^{-\epsilon r T} .
$$

1093 A bound on the event rate $R_{C}$ at confidence level $C$ is 1094 then given by demanding the probability of observing at 1095 least one event $1-e^{-\epsilon R_{C} T} \geq C$, likewise, one would 1096 expect to observe event rates

$$
\frac{g_{F, j} B_{j}}{\sigma_{j} \eta_{j}}=\frac{4}{\mu_{B}} m_{a} \xi \equiv \mathcal{B}_{\mathrm{p}},
$$

$$
r \geq R_{C} \equiv \frac{-\log (1-C)}{\epsilon T} .
$$

for $\xi=f_{\mathrm{SB}},{ }_{1097}$ The physical parameter space of the ALPs is con1052 for $\xi \equiv \frac{f_{\mathrm{B}}}{f_{\text {int }}}, g_{F, j}$ being the $g$-factor for the magnetometer ${ }_{1098}$ strained by demanding that $r \geq R_{C}$. Similar arguments $1053 j$, and $\left\|S_{(i)}\right\|=1 / 2$ since we only consider coupling to 1099 for defining constraints can be found, e.g., Ref. [40]. The 1054 spin- $1 / 2$ particles. Here, the normalization is such that $\mathcal{B}_{\mathrm{p}}{ }_{1100}$ total time that the network is sensitive to the measur1055 is the same for all magnetometers, though each individual 1101 able parameters, $\tilde{T}\left(\Delta t, \mathcal{B}_{\mathrm{p}}^{\prime}\right)$, may be less than the total 1056 sensor may observe a different pseudo-magnetic field, $B_{j} .{ }_{1102}$ measurement time. These parameters are related to the ${ }_{1057}$ There are two factors that must be considered when ${ }_{1103}$ physical parameters via Eq. (A.5) and Eq. (A.10). One 1058 determining if axion domain walls are observable by the ${ }_{1104}$ finds a sensitivity bound for $f_{\text {int }}$ in terms of $m_{a}$ and $\xi$, 1059 network: the magnitude of the signal $\mathcal{B}_{\mathrm{p}}$ and the rate of 1060 signals. Domain walls are assumed to exist in a static 1105 1061 (or virialized) network across the galaxy through which 1062 Earth traverses. For a domain-wall velocity $v$, the du1063 ration of a signal is $\Delta t=\Delta x / v$. Filters and bandwidth ${ }_{1107}^{1106}$ 1064 limitations generally reduce the magnitude by a factor 1065 dependent on the signal duration, which affects the sen1066 sitivity of the network (see Appendix in Ref. [29]).

${ }_{1067}$ Meanwhile, if the domain walls induce a strong enough 1068 signal to be observed, but are so infrequent that one is 1069 unlikely to be found over the course of a measurement, 1070 then the network is effectively insensitive. If the energy 1071 density of domain walls across the galaxy is $\rho_{\mathrm{DW}}$, then 1072 the average rate of domain walls passing through Earth 1073 is given by

$$
\begin{aligned}
f_{\text {int }} & \leq \frac{1}{\xi} \sqrt{\frac{-\bar{v} \rho_{\mathrm{DW}} \epsilon}{8 m_{a} \log (1-C)} \tilde{T}\left(\Delta t, \mathcal{B}_{\mathrm{p}}^{\prime}\right)} \\
\text { for } \Delta t & =\frac{2 \sqrt{2}}{\bar{v} m_{a}} \text { and } \mathcal{B}_{\mathrm{p}}^{\prime}=\frac{4 m_{a} \xi}{\mu_{B} \zeta},
\end{aligned}
$$

${ }_{1108}$ where $\mathcal{B}_{\mathrm{p}}^{\prime}$ is the sensitivity of the network and $\zeta$ is the 1109 magnitude-to-noise ratio induced by a signal with mag1110 nitude $\mathcal{B}_{\mathrm{p}}$. The main calculation from the network data 1111 needed for this sensitivity bound is $\tilde{T}$. This is calculated 1112 by measuring the sensitivity of the network over time 1113 for different signal durations $\Delta t$ and integrating over the 1114 time during which the network is sensitive to $\mathcal{B}_{\mathrm{p}}=\zeta \mathcal{B}_{\mathrm{p}}^{\prime}$. 1115 Finally, if, after analyzing the data, no domain walls are 1116 found, Eq. (A.13) defines an exclusion region.

$$
r=\frac{\bar{v} \rho_{\mathrm{DW}}}{\sigma_{\mathrm{DW}}}=\frac{\bar{v} \rho_{\mathrm{DW}}}{8 m_{a} f_{\mathrm{SB}}^{2}}
$$

Appendix B: Conversion between magnetic field 1118 units and proton spin coupling

1074 where $\bar{v}$ is the typical relative speed.

${ }_{1075}$ The physical parameters describing the ALP domain ${ }_{1119}$ The amplitude of a signal appearing in the magnetic 1076 walls $\left(m_{a}, f_{\mathrm{SB}}\right.$, and $\left.f_{\text {int }}\right)$ must be related to the parame- 1120 field data from a GNOME magnetometer due to interac1077 ters observable by the network $\left(\mathcal{B}_{\mathrm{p}}\right.$ and $\left.\Delta t\right)$. The energy ${ }_{1121}$ tion of atomic spins with an ALP field $a$ via the linear 
1122 coupling described by Eq. (1) varies based on the atomic 1170 The above relations define the fractional spin polariza1123 species. In every GNOME magnetometer, the atomic va- 1171 tion of the nucleus relative to the spin polarization of the 1124 por cell is located within a multi-layer magnetic shield 1172 atom:

1125 made of mu-metal and, in some cases a ferrite inner1126 most layer. Interactions of the ALP field with electron 1127 spins in the magnetic shielding material can generate a 1128 compensating magnetic field that could partially cancel 1129 the energy shift due to the interaction of the ALP field 1130 with atomic electrons in the vapor cell, as discussed in 1131 Ref. [27]. For this reason, GNOME magnetometers are 1132 most sensitive to interactions of the ALP field with nu1133 clear spins.

$$
\sigma_{N, F} \equiv \frac{\langle\mathbf{I} \cdot \mathbf{F}\rangle}{F(F+1)} .
$$

1173 The next step is to relate $\sigma_{N, F}$ to the spin polarization 174 of the valence proton $\sigma_{p, F}$ for a particular $F$. As dis1175 cussed in Ref. [28], a reasonable estimate for $\mathrm{K}, \mathrm{Rb}$, and ${ }_{1176}$ Cs nuclei can be obtained from the nuclear shell model 1177 by assuming that the nuclear spin $\mathbf{I}$ is due to the or1178 bital motion and intrinsic spin of only the valence nu1179 cleon and that the spin and orbital angular momenta of 1180 all other nucleons sum to zero. This is the assumption of 1181 the Schmidt or single-particle model [54]. In the Schmidt 1182 model, the nuclear spin $\mathbf{I}$ is generated by a combination 1135 All GNOME magnetometers active during Science ${ }_{1136}$ Run 2 measure spin-dependent interactions of alkali 1137 atoms whose nuclei have valence protons. Thus the ${ }_{1138}$ GNOME is primarily sensitive to spin-dependent interac1139 tions of ALP fields with proton spins. Consequently, the 1140 expected signal amplitude measured by a GNOME mag1141 netometer due to the pseudo-magnetic field pulse from ${ }^{1186}$ 1142 passage of Earth through an ALP domain wall must 1143 be rescaled by the ratio of the proton spin content of 1188 1144 the probed ground-state hyperfine level(s) to their gyro1145 magnetic ratio. Some GNOME magnetometers optically ${ }_{1146}$ pump and probe a single ground-state hyperfine level, 1147 while others rely on the technique of spin-exchange re1148 laxation free (SERF) magnetometry in which the spin1149 exchange collision rate is much faster than the Larmor 1150 precession frequency $[49,52,53]$. For SERF magnetome1151 ters a weighted average of the ground-state Zeeman sub1152 levels over both ground-state hyperfine levels is optically 1153 pumped and probed.

${ }_{1154}$ Table II shows the relevant factors needed to convert 1155 the magnetic field signal recorded by GNOME magne1156 tometers into the expected pseudo-magnetic field due to 1157 interaction of an ALP field with the proton spin. Detailed 1158 calculations are carried out in Ref. [28]. The relationship 1159 of the expectation value for total atomic angular momen1160 tum $\langle\mathbf{F}\rangle$ to the nuclear spin $\langle\mathbf{I}\rangle$ can be estimated based 1161 on the Russell-Saunders $L S$-coupling scheme:

1162

$$
\begin{aligned}
\langle\mathbf{F}\rangle & =\left\langle\mathbf{S}_{e}\right\rangle+\langle\mathbf{L}\rangle+\langle\mathbf{I}\rangle, \\
& =\frac{\left\langle\mathbf{S}_{e} \cdot \mathbf{F}\right\rangle}{F(F+1)}\langle\mathbf{F}\rangle+\frac{\langle\mathbf{L} \cdot \mathbf{F}\rangle}{F(F+1)}\langle\mathbf{F}\rangle+\frac{\langle\mathbf{I} \cdot \mathbf{F}\rangle}{F(F+1)}\langle\mathbf{F}\rangle, \quad{ }_{1207}
\end{aligned}
$$

$$
\begin{aligned}
\sigma_{p, F} & =\frac{\left\langle\mathbf{S}_{p} \cdot \mathbf{I}\right\rangle}{I(I+1)} \sigma_{N, F} \\
& =\frac{S_{p}\left(S_{p}+1\right)+I(I+1)-\ell(\ell+1)}{2 I(I+1)} \sigma_{N, F} \\
& =\sigma_{p} \sigma_{N, F},
\end{aligned}
$$

1189 where it is assumed that the valence nucleon is in a well190 defined state of $\ell$ and $S_{p}$, and $\sigma_{p}$ is defined to be the frac191 tional proton spin polarization for a given nucleus [28].

For comparison between GNOME magnetometers us193 ing different atomic species, it is essential to evaluate the 1194 uncertainty in the estimate of $\sigma_{p, F}$ based on the Schmidt 1195 model. To estimate this uncertainty, we compare calcula1196 tions of $\sigma_{p, F}$ based on the Schmidt model to the results of ${ }_{1197}$ the semi-empirical calculations described in Refs. $[55,56]$ 1198 and to the results of detailed nuclear shell-model calcula1199 tions where available [57-59]. Conservatively, we assign 1200 the uncertainty in $\sigma_{p, F}$ to be given by the full range (max1201 imum to minimum) of the values of $\sigma_{p, F}$ calculated by 1202 these various methods. It turns out that in each consid1203 ered case, the estimate based on the Schmidt model gives 1204 the largest value of $\left|\sigma_{p, F}\right|$, causing the theoretical uncer1205 tainties in estimates of $\sigma_{p, F}$ to be one-sided as shown in ${ }_{1206}$ Table II. Further details are discussed in Ref. [28].

\section{SERF magnetometers}

1164

${ }_{1208}$ SERF magnetometers operate in a regime where the ${ }_{1165}$ where $\mathbf{S}_{e}$ is the electronic spin and $\mathbf{L}$ is the orbital an- ${ }_{1209}$ Larmor frequency is small compared to the spin-exchange 1166 gular momentum. GNOME magnetometers pump and ${ }_{1210}$ rate, so that the rapid spin-exchange locks together the ${ }_{1167}$ probe atomic states with $L=0$, which simplifies the ${ }_{1211}$ expectation values of the angular momentum projection 1168 above equation to:

$$
\langle\mathbf{F}\rangle=\frac{\left\langle\mathbf{S}_{e} \cdot \mathbf{F}\right\rangle}{F(F+1)}\langle\mathbf{F}\rangle+\frac{\langle\mathbf{I} \cdot \mathbf{F}\rangle}{F(F+1)}\langle\mathbf{F}\rangle .
$$
${ }_{1212}\left\langle M_{F}\right\rangle$ in both ground-state hyperfine levels of the alkali 1213 atom. Because the Landé $g$-factors $g_{F}$ in the two ground-

(B.2) ${ }_{1214}$ state hyperfine levels have nearly equal magnitudes but 1215 opposite signs, the magnitude of the effective Landé $g$ 1216 factor in a SERF magnetometer, $\langle g\rangle_{\mathrm{hf}}$, is reduced com1217 pared to that in optical atomic magnetometers where a

$$
\langle\mathbf{I} \cdot \mathbf{F}\rangle=\frac{1}{2}\left[F(F+1)+I(I+1)-S_{e}\left(S_{e}+1\right)\right] .
$$

(B.3) ${ }_{1218}$ single ground-state hyperfine level is probed. 
Table II. Fractional proton spin polarization $\sigma_{p, F}$, Landé $g$-factors $g_{F}$, and their ratios for the ground state hyperfine levels used in GNOME, and the weighted average of these values across both hyperfine levels $\left(\left\langle\sigma_{p}\right\rangle_{\mathrm{hf}} /\langle g\rangle_{\mathrm{hf}}\right)$ applicable to SERF magnetometers in the low-spin-polarization limit. The estimates are based on the single-particle Schmidt model for nuclear spin [54] and the Russell-Saunders scheme for the atomic states. Uncertainties in the values for $\sigma_{p, F}$ describe the range of different results from calculations based on the Schmidt model, semi-empirical models [55, 56], and large-scale nuclear shell model calculations where available [57-59]. The uncertainties in $\sigma_{p, F}$ and $\left\langle\sigma_{p}\right\rangle_{\mathrm{hf}}$ are one-sided because alternative methods to the Schmidt model generally predict smaller absolute values of the proton spin polarization. See Ref. [28] for further details.

\begin{tabular}{lrrrr}
\hline \hline Atom (state) & $\sigma_{p, F}$ & $g_{F}$ & $\sigma_{p, F} / g_{F}$ & $\left\langle\sigma_{p}\right\rangle_{\mathrm{hf}} /\langle g\rangle_{\mathrm{hf}}$ \\
\hline${ }^{39} \mathrm{~K}(F=2)$ & $-0.15_{-0.00}^{+0.06}$ & 0.50 & $-0.30_{-0.00}^{+0.12}$ & $-0.5_{-0.0}^{+0.2}$ \\
${ }^{39} \mathrm{~K}(F=1)$ & $-0.25_{-0.00}^{+0.10}$ & -0.50 & $0.50_{-0.19}^{+0.00}$ & \\
${ }^{85} \mathrm{Rb}(F=3)$ & $-0.12_{-0.00}^{+0.02}$ & 0.33 & $-0.36_{-0.00}^{+0.05}$ & $-0.8_{-0.0}^{+0.1}$ \\
${ }^{85} \mathrm{Rb}(F=2)$ & $-0.17_{-0.00}^{+0.02}$ & -0.33 & $0.50_{-0.07}^{+0.00}$ & \\
${ }^{87} \mathrm{Rb}(F=2)$ & $0.25_{-0.05}^{+0.00}$ & 0.50 & $0.50_{-0.11}^{+0.00}$ & $0.8_{-0.2}^{+0.0}$ \\
${ }^{87} \mathrm{Rb}(F=1)$ & $0.42_{-0.09}^{+0.00}-0.50-0.83_{-0.00}^{+0.18}$ & \\
${ }^{133} \mathrm{Cs}(F=4)$ & $-0.10_{-0.00}^{+0.05}$ & 0.25 & $-0.39_{-0.00}^{+0.19}$ & $-1.2_{-0.0}^{+0.6}$ \\
${ }^{133} \mathrm{Cs}(F=3)$ & $-0.13_{-0.00}^{+0.06}-0.25$ & $0.50_{-0.24}^{+0.00}$ & \\
\hline \hline
\end{tabular}

${ }_{1219}$ To calculate the effective Landé $g$-factor averaged over ${ }_{1236}$ Substituting the above expressions into Eq. (B.6) yields 1220 hyperfine levels, $\langle g\rangle_{\mathrm{hf}}$, for a SERF magnetometer, it is 1221 instructive to consider the equation describing the mag1222 netic torque on an alkali atom,

$$
\langle g\rangle_{\mathrm{hf}} \mu_{B} \mathbf{B} \times \boldsymbol{\beta} \approx \frac{d \boldsymbol{\beta}}{d t},
$$

$$
g_{s} \mu_{B} \mathbf{B} \times\left\langle\mathbf{S}_{e}\right\rangle \approx \frac{d\langle\mathbf{F}\rangle}{d t},
$$

1237 where

1223 where $g_{s} \approx 2$ is the electron $g$-factor and we have ig1224 nored the contribution of the nuclear magnetic moment. ${ }_{1225}$ In the SERF regime, where the alkali vapor is in spin1226 exchange equilibrium, the populations of the Zeeman 1227 sublevels correspond to the spin-temperature distribu1228 tion [60] described by a density matrix in the Zeeman 1229 basis given by $[61,62]$

$$
\boldsymbol{\rho}=C e^{\boldsymbol{\beta} \cdot \mathbf{F}},
$$

$$
\langle g\rangle_{\mathrm{hf}}=\frac{3 g_{s}}{3+4 I(I+1)} .
$$

${ }_{1238}$ Equation (B.13) can be compared to the $g$-factor for a 1239 particular alkali ground-state hyperfine level [63],

$$
g_{F}= \pm \frac{g_{s}}{2 I+1}
$$

${ }_{1240}$ The effective proton spin polarization $\left\langle\sigma_{p}\right\rangle_{\mathrm{hf}}$ for SERF (B.7) ${ }_{1241}$ magnetometers can also be derived by considering the 1242 relevant torque equation

${ }_{1230}$ where $C$ is a normalization constant and $\boldsymbol{\beta}$ is the spin1231 temperature vector defined to point in the direction of 1232 the spin polarization $P$ with magnitude

$$
\begin{aligned}
\left(\frac{1}{f_{\mathrm{int}}} \nabla a\right) \times \frac{\left\langle\mathbf{S}_{p}\right\rangle}{\left\|S_{p}\right\|} & =\frac{d\langle\mathbf{F}\rangle}{d t}, \\
\left(\frac{1}{f_{\mathrm{int}}} \nabla a\right) \times \frac{\sigma_{p}}{\left\|S_{p}\right\|}\langle\mathbf{I}\rangle & =\frac{d\langle\mathbf{F}\rangle}{d t},
\end{aligned}
$$$$
\beta=\ln \left(\frac{1+P}{1-P}\right)
$$

1233 In the low-spin-polarization limit,

$$
\boldsymbol{\rho} \approx C(1+\boldsymbol{\beta} \cdot \mathbf{F})
$$

1246 where we have used the fact that GNOME as configured 1247 for Science Run 2 is sensitive to the coupling of the ALP (B.9) 1248 field to proton spins. In the low-spin-polarization limit, 1249 based on Eqs. (B.10) and (B.11),

1234 If follows that

$$
\left\langle\mathbf{S}_{e}\right\rangle=\operatorname{Tr}\left(\boldsymbol{\rho} \mathbf{S}_{e}\right)=\frac{1}{3} S_{e}\left(S_{e}+1\right) \boldsymbol{\beta}=\frac{1}{4} \boldsymbol{\beta},
$$

$$
\left(\frac{1}{f_{\mathrm{int}}} \nabla a\right) \times \frac{\left\langle\sigma_{p}\right\rangle_{\mathrm{hf}}}{\left\|S_{p}\right\|} \boldsymbol{\beta}=\frac{d \boldsymbol{\beta}}{d t},
$$

1235 and

$$
\langle\mathbf{I}\rangle=\operatorname{Tr}(\boldsymbol{\rho} \mathbf{I})=\frac{1}{3} I(I+1) \boldsymbol{\beta}
$$

1250 where

$$
\left\langle\sigma_{p}\right\rangle_{\mathrm{hf}}=\sigma_{p} \frac{4 I(I+1)}{3+4 I(I+1)} .
$$


${ }_{1251}$ Table II shows the ratio between the effective proton 1275 ing into account the relative abundances of the differ1252 spin polarization averaged over both ground-state hyper- 1276 ent atomic species at the cell temperature of $\approx 150^{\circ} \mathrm{C}$ ${ }_{1253}$ fine levels, $\left\langle\sigma_{p}\right\rangle_{\mathrm{hf}}$, to the effective Landé $g$-factor, $\langle g\rangle_{\mathrm{hf}},{ }_{1277}\left(\approx 9 \%{ }^{39} \mathrm{~K}, \approx 65.5 \%{ }^{85} \mathrm{Rb}, \approx 25.5 \%{ }^{87} \mathrm{Rb}\right)$, we find 1254 in the low-spin-polarization regime. Note that the mag- ${ }_{1278}$ that $\langle g\rangle_{\mathrm{hf}} \approx 0.193,\left\langle\sigma_{p}\right\rangle_{\mathrm{hf}}=-0.073_{-0.000}^{+0.010}$, and thus 1255 nitudes of $\left\langle\sigma_{p}\right\rangle_{\mathrm{hf}} /\langle g\rangle_{\mathrm{hf}}$ are in general similar or slightly ${ }_{1279}\left\langle\sigma_{p}\right\rangle_{\mathrm{hf}} /\langle g\rangle_{\mathrm{hf}}=-0.38_{-0.00}^{+0.05}$ for the Hefei magnetometer. 1256 larger than the magnitudes of $\sigma_{p, F} / g_{F}$ for a single hy1257 perfine level.

${ }_{1258}$ To determine the actual values of $\left\langle\sigma_{p}\right\rangle_{\mathrm{hf}} /\langle g\rangle_{\mathrm{hf}}$ for the ${ }_{1280}$ 1259 SERF magnetometers used in GNOME's Science Run 2, 1260 more detailed considerations are required.

1281 The Lewisburg GNOME station employs a SERF mag${ }_{1261}$ The values for the ratio of the effective proton spin ${ }_{1282}$ netometer in a closed-loop, two-beam configuration. The 1262 polarization to the effective Landé $g$-factors for each ${ }_{1283}$ vapor cell contains only ${ }^{87} \mathrm{Rb}$ atoms. The Lewisburg ${ }_{1263}$ GNOME magnetometer active during Science Run 2 are ${ }_{1284}$ SERF magnetometer operates with a spin-polarization 1264 given in Table I. ${ }_{1285} P \approx 0.5$, outside the low-spin-polarization regime. Dis1286 cussions of the high-polarization regime are given in ${ }_{1287}$ Refs. [62, 64]. For a nucleus with $I=3 / 2$, the effective 1265 a. Hefei magnetometer 1288 Landé $g$-factor is given by

1266 The Hefei GNOME station employs a SERF mag1267 netometer in a closed-loop, single-beam configuration, 1268 where the laser light is resonant with the Rb D1 line ${ }_{1269}$ (pressure-broadened by 600 torr of nitrogen gas to a 1270 linewidth of $\sim 10 \mathrm{GHz}$ ). The Hefei SERF magnetome1271 ter operates in the low-spin-polarization mode. The va1272 por cell contains ${ }^{39} \mathrm{~K},{ }^{85} \mathrm{Rb}$, and ${ }^{87} \mathrm{Rb}$ atoms in natural

$$
\langle g\rangle_{\mathrm{hf}}=g_{s} \frac{1+P^{2}}{6+2 P^{2}},
$$
1289 and the effective proton spin polarization is given by

$$
\left\langle\sigma_{p}\right\rangle_{\mathrm{hf}}=\sigma_{p} \frac{5+P^{2}}{6+2 P^{2}} .
$$

1273 abundance, so spin-exchange collisions average over both 1290 Based on Eqs. (B.18) and (B.19), we find that for the 1274 ground-state hyperfine levels of all three species. Tak- ${ }_{1291}$ Lewisburg magnetometer $\left\langle\sigma_{p}\right\rangle_{\mathrm{hf}} /\langle g\rangle_{\mathrm{hf}}=0.70_{-0.15}^{+0.00}$. 


\section{Figures}

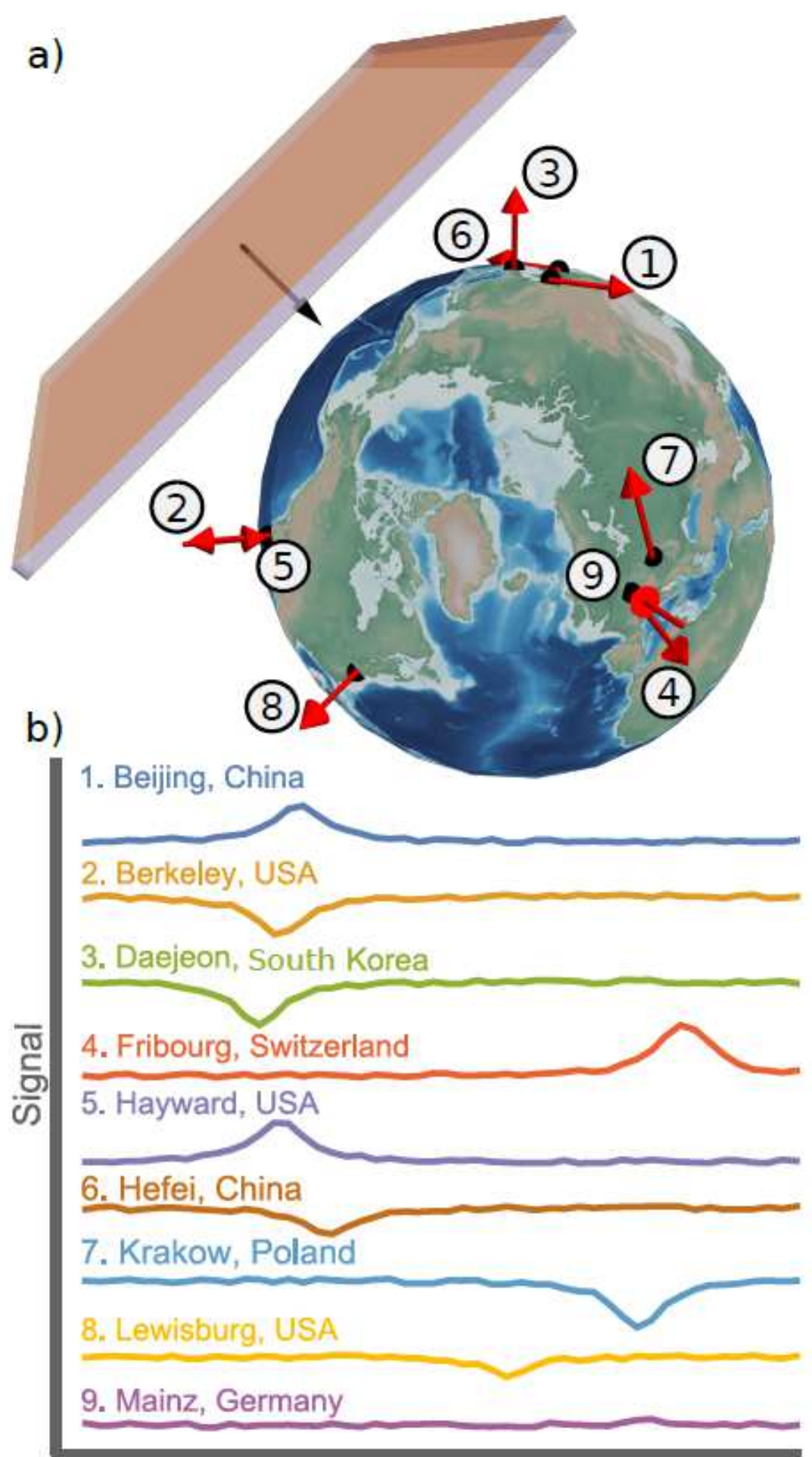

Time

Figure 1

(a) Visualization of an ALP domain wall crossing the Earth. The red arrows indicate the position and sensitive axes of the GNOME magnetometers during Science Run 2 (Sec. I). (b) Simulation of the signals expected to be observed from a domain-wall crossing. Note: The designations employed and the 
presentation of the material on this map do not imply the expression of any opinion whatsoever on the part of Research Square concerning the legal status of any country, territory, city or area or of its authorities, or concerning the delimitation of its frontiers or boundaries. This map has been provided by the authors.

Significance $(\sigma)$

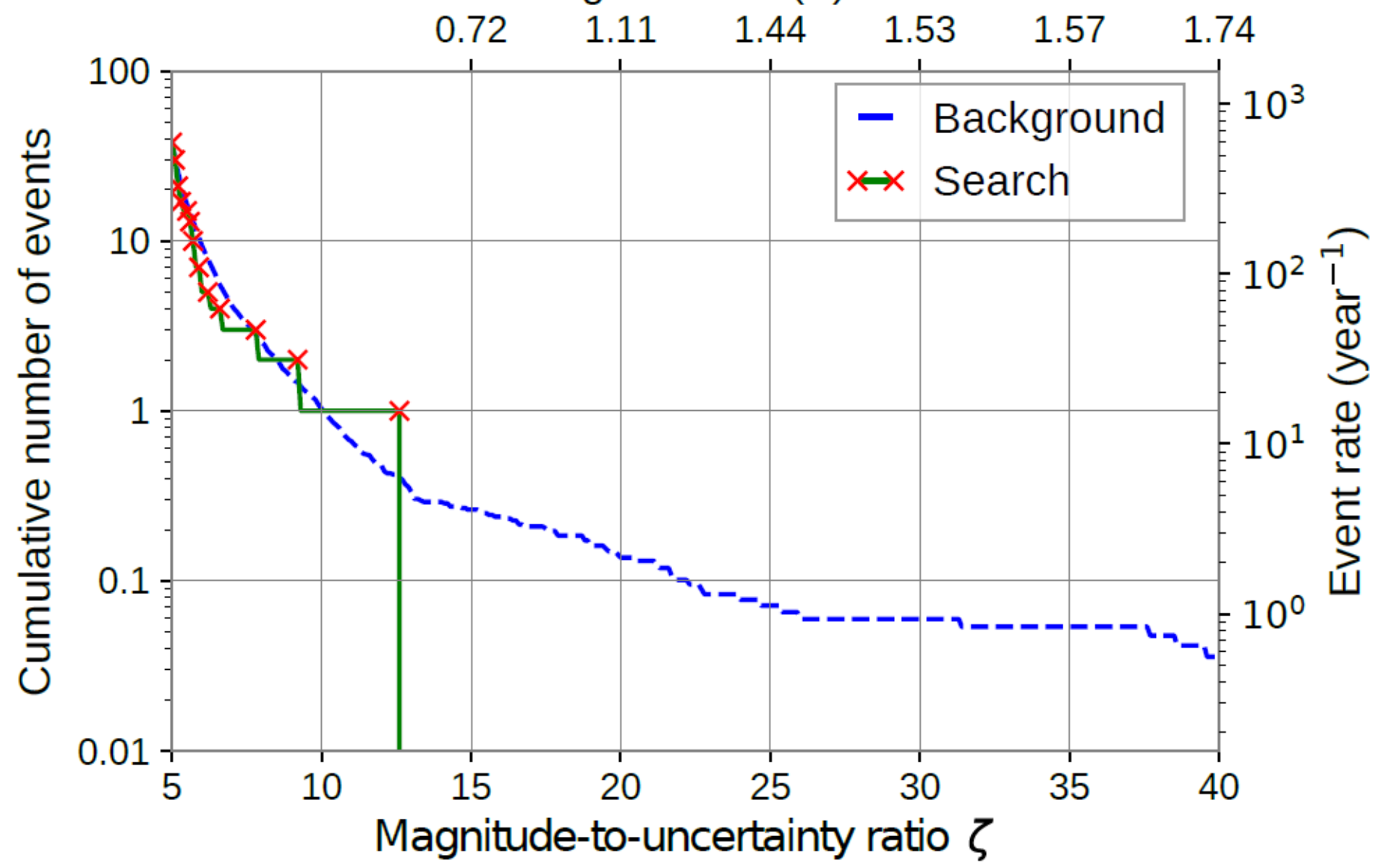

Figure 2

The blue dashed line represents the number events expected from the background in the twenty-three days of data from Science Run 2. 10.7 years of time-shuffled data are used to evaluate the background. The solid green line represents the number of events measured in Science Run 2. The red crosses indicate at which magnitude-to-uncertainty ratio new events are found. The upper axis indicates the statistical significance in units of Gaussian standard deviations of finding one event in the search data. The event with greatest magnitude-to-uncertainty ratio is found at 12.6 . 


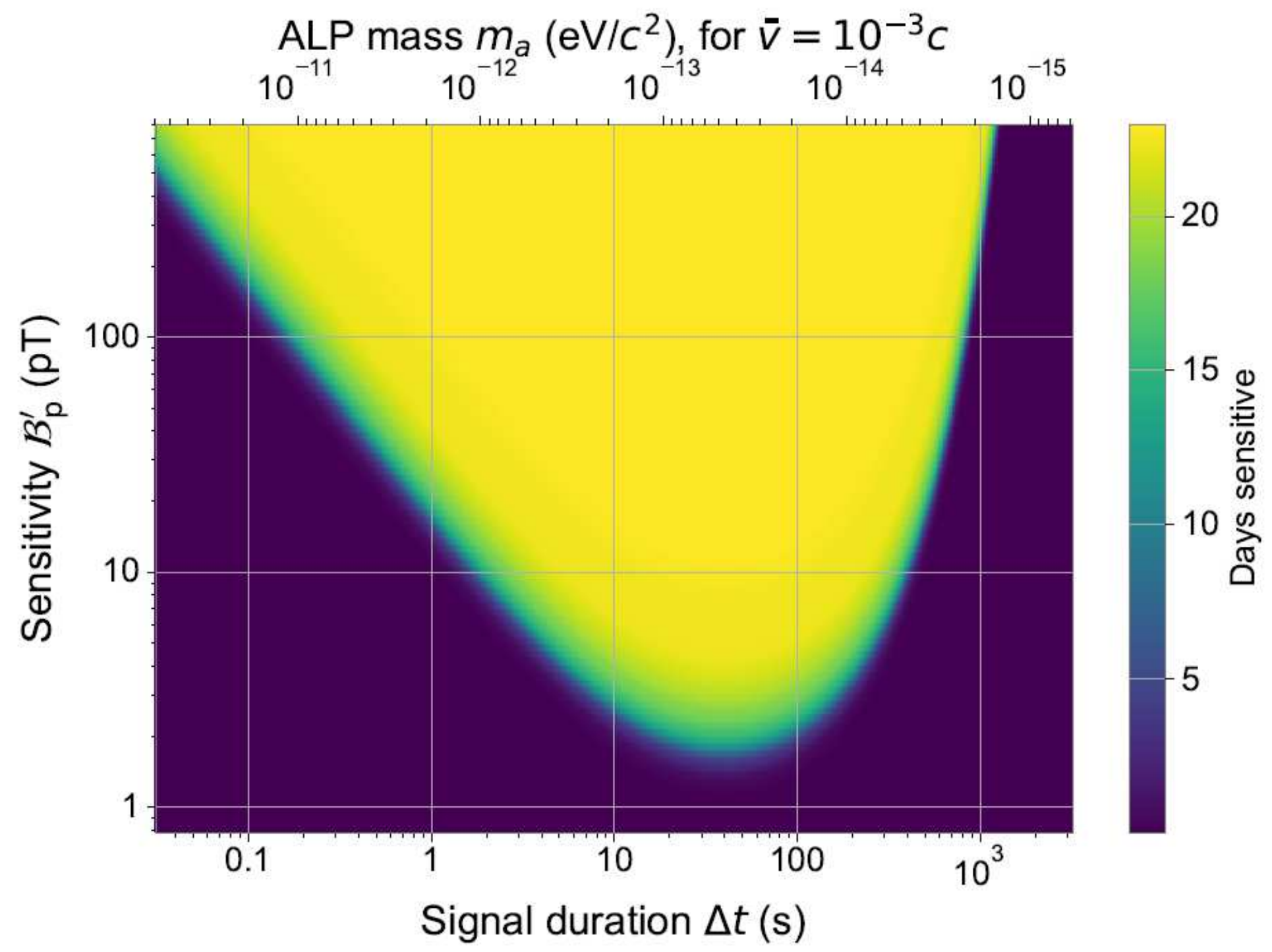

Figure 3

Amount of time $T^{\sim}$ that the GNOME is sensitive to domain walls with a given duration $\Delta t$ and normalized pseudo-magnetic field magnitude sensitivity B'p throughout Science Run 2, the magnitude of an event needed to induce a signal with a magnitude-to-uncertainty ratio of one [see Eq. (11)]. Only the worst-case direction is considered. The plot assumes the parameters of the analysis: $20 \mathrm{~s}$ averaging time, $1.67 \mathrm{mHz}$ first-order zero-phase Butterworth filter, and $50 \mathrm{~Hz}$ and $60 \mathrm{~Hz}$ zero-phase notch filters with a Q-factor of 60. 
a)

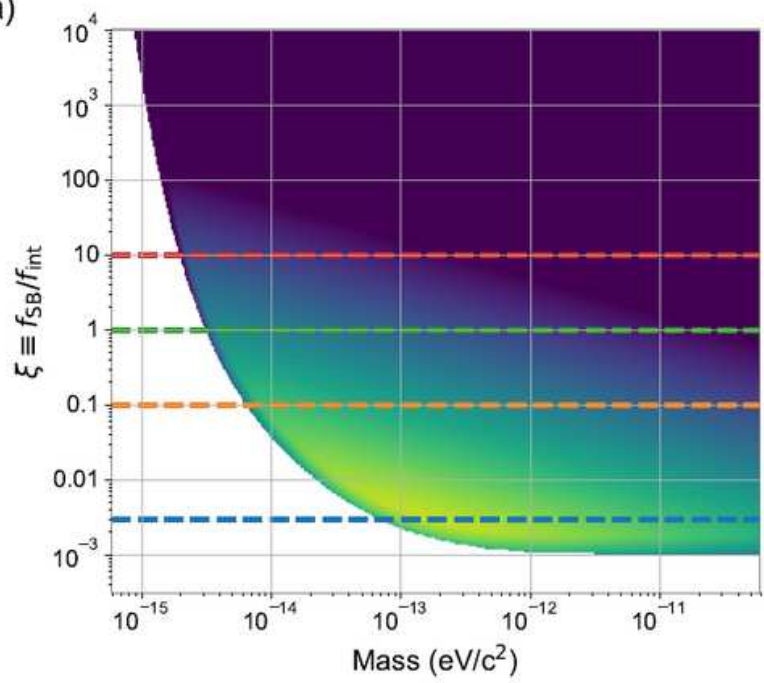

b)

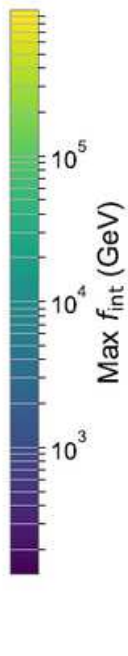

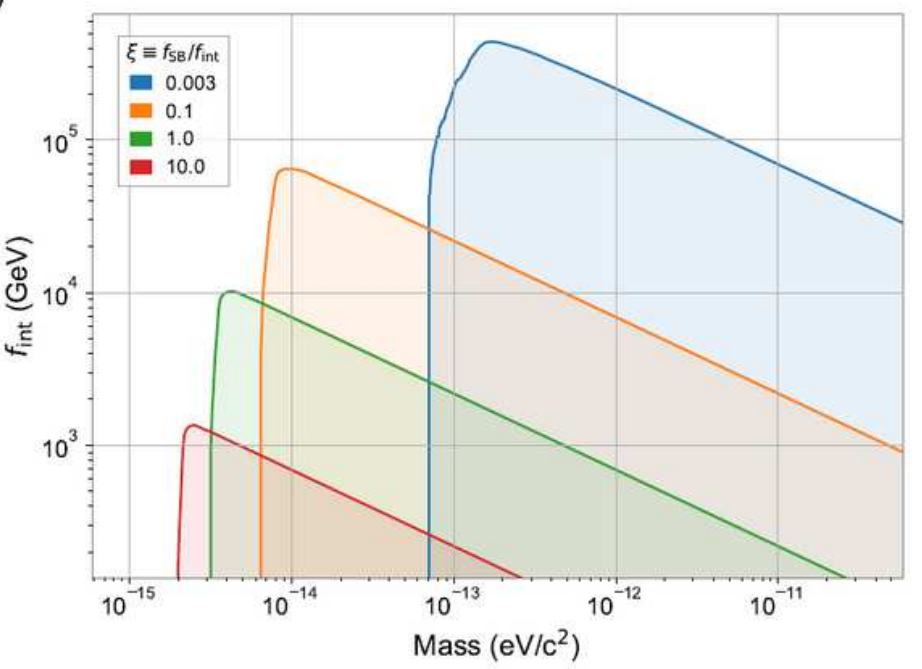

Figure 4

Parameter space of the ALP constrained by the presented analysis of Science Run 2 with $90 \%$ confidence level (see Sec. II). The relationship between ALP theory parameters and measured quantities are discussed in Sec. III and Appendix A.(a) In color, upper bound on the the interaction scale, fint, to which the GNOME was sensitive as a function of ma and the ratio $\xi \otimes \mathrm{fSB} /$ fint. (b) Cross-sections of the excluded parameter volume in (a) for different ratios $\xi$ represented by the corresponding dashed lines of the matching color in (a).

a)

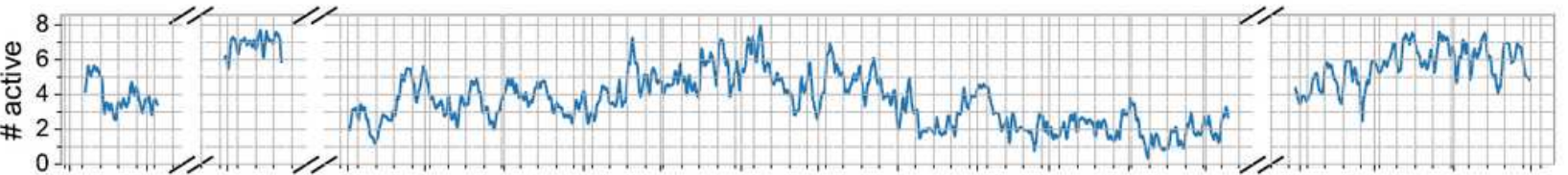

b)

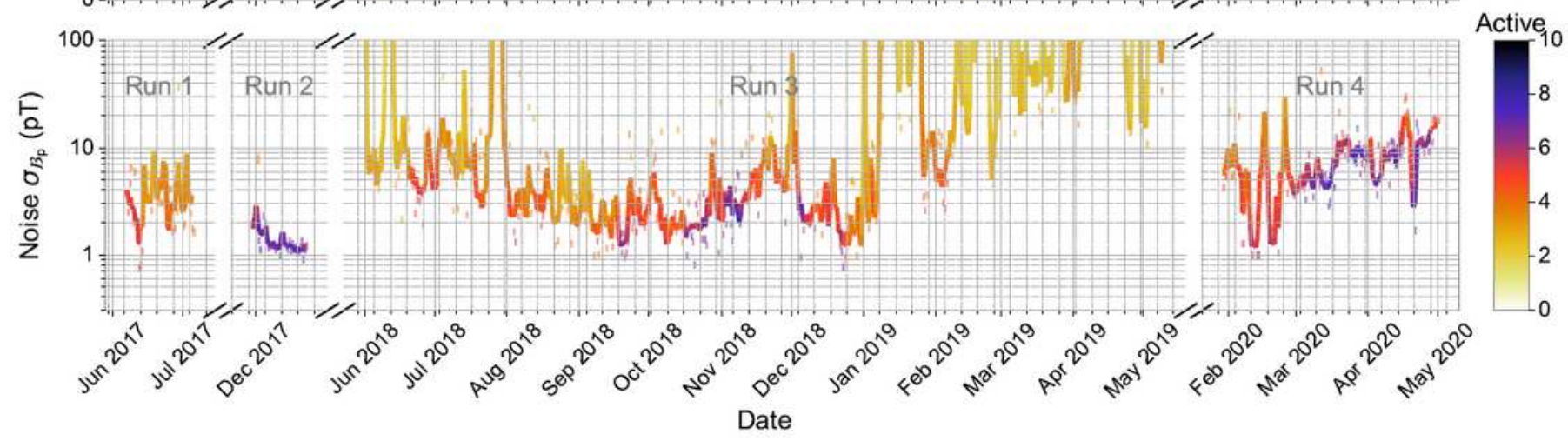

\section{Figure 5}

Summary of the GNOME performance during the four Science Runs from 2017 to 2020. The raw magnetometer data are averaged for $20 \mathrm{~s}$ and their standard deviation is calculated over a minimum of one and a maximum of two hours segments depending on the availability of continuous data segments. For each binned point, the combined network noise considering the worse case domain-wall crossing direction is evaluated as defined in Ref. [29]. (a) One-day rolling average of the number of active sensors. 
(b) Multi-colored solid line represents the one-day rolling average of the combined network noise and the multi-colored dashes show the noise of the individual sampled segments. The data are preprocessed with the same filters used for the analysis. The number of magnetometers active is indicated by the color of the line and dashes.

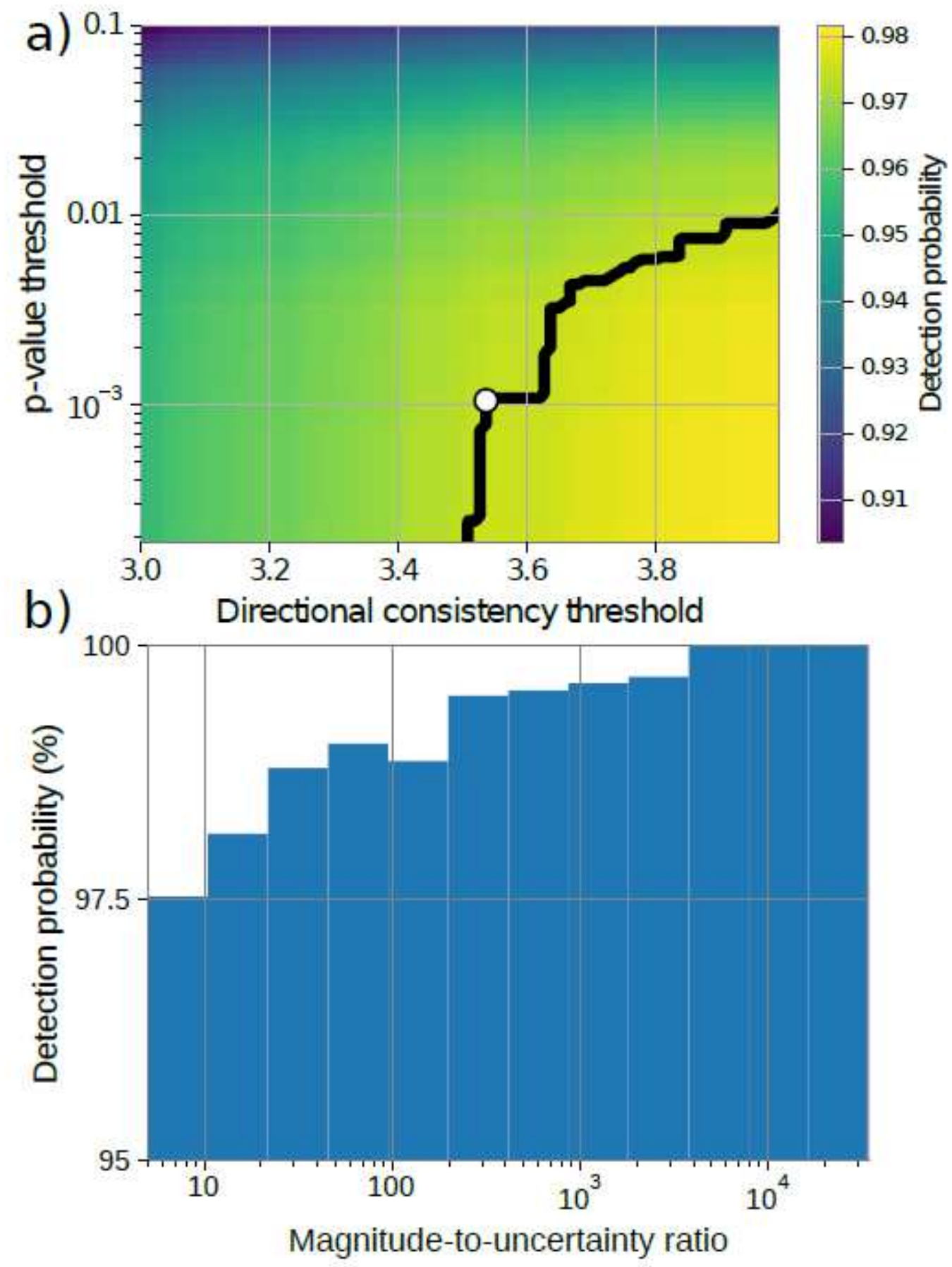

Figure 6

Summary of the true-positive analysis results.(a) shows the probability of detecting a domain-wallcrossing event with randomized parameters (as discussed in the text) as a function of $p$-value and directional-consistency thresholds. The inserted events have a magnitude-to-uncertainty ratio between 5 
and 10. The black line indicates the com-bination of parameters corresponding to a $97.5 \%$ detection probability. The white dot indicates the particular thresholds chosen for the analysis. (b) Shows the mean detection probability reached for different magnitude-to-uncertainty ratios for the chosen thresholds. 